\title{
Electron-vibration interaction in transport through atomic gold wires
}

\author{
J. K. Viljas, ${ }^{1}$ J. C. Cuevas, ${ }^{1,2}$ F. Pauly, ${ }^{1}$ and M. Häfner ${ }^{1}$ \\ ${ }^{1}$ Institut für Theoretische Festkörperphysik, Universität Karlsruhe, 76128 Karlsruhe, Germany \\ ${ }^{2}$ Departamento de Fúsica Teórica de la Materia Condensada, \\ Universidad Autónoma de Madrid, 28049-Madrid, Spain
}

(Dated: February 2, 2008)

\begin{abstract}
We calculate the effect of electron-vibration coupling on conduction through atomic gold wires, which was measured in the experiments of Agrait et al. [Phys. Rev. Lett. 88, 216803 (2002)]. The vibrational modes, the coupling constants, and the inelastic transport are all calculated using a tight-binding parametrization and the non-equilibrium Green function formalism. The electronvibration coupling gives rise to small drops in the conductance at voltages corresponding to energies of some of the vibrational modes. We study systematically how the position and height of these steps vary as a linear wire is stretched and more atoms are added to it, and find a good agreement with the experiments. We also consider two different types of geometries, which are found to yield qualitatively similar results. In contrast to previous calculations, we find that typically there are several close-lying drops due to different longitudinal modes. In the experiments, only a single drop is usually visible, but its width is too large to be accounted for by temperature. Therefore, to explain the experimental results, we find it necessary to introduce a finite broadening to the vibrational modes, which makes the separate drops merge into a single, wide one. In addition, we predict how the signatures of vibrational modes in the conductance curves differ between linear and zigzag-type wires.
\end{abstract}

PACS numbers: 72.10.Di, 73.23.-b, 73.40.Jn

\section{INTRODUCTION}

The electron transport properties of atomic point contacts between two metallic electrodes have been intensively studied during the past decade [1]. Contacts of this type are typically formed by using mechanically controllable break junctions (MCBJ) or with the tip of a scanning tunneling microscope (STM). It has been found that the conductance of such contacts depends strongly on the electronic structure of the metals, and for monovalent metals there is a tendency for quantization in units of the conductance quantum $G_{0}=2 e^{2} / h[2]$. Point contacts formed from Gold $(\mathrm{Au})$, Platinum $(\mathrm{Pt})$, or Iridium ( $\mathrm{Ir}$ ) by one of the MCBJ or STM methods have the further interesting property of being able to sustain single-atom thick chains, so called atomic wires 3, 4, 5]. Following their discovery, a good amount of experimental [6, 7] and theoretical 8, 9, 10, 11, 12, 13] work has been carried out to further investigate the conduction properties of atomic wires. It is by now well established that the zerobias conductance of gold wires is close to one $G_{0}$ due to a single, almost fully open transmission channel at the Fermi energy 2, 14, 15, 16. However, less detailed work has been done in the study of truly nonequilibrium properties, such as the current-voltage characteristics 17].

In recent experiments, the conductance vs. voltage characteristics $G(V)$ of gold wires formed by the STM technique were measured [18]. It was observed that the conductance often has a very pronounced, single drop from $G_{0}$ at a critical voltage $V_{p h}=10-20 \mathrm{mV}$, marking the onset of a dissipative process. The size of the drop was on the order of $0.5 \%-2.0 \%$ of $G_{0}$. It was also found that stretching of the wire typically leads to an increase in the step, and to a decrease in the critical voltage $V_{p h}$. Based on simple arguments for infinite single-orbital tight-binding chains, these findings were interpreted as a sign of the excitation of vibrational modes in the wire: only a single longitudinal mode with twice the Fermi wave vector can be excited, since this corresponds to the momentum which must be transferred from an electron to the vibrations in a single backscattering process. Although the validity of such arguments for a wire of finite length (of typically less than 10 atoms) can be questioned, the interpretation was backed up by firstprinciples calculations [19, 20]. The authors of Ref. 19 emphasize the importance of so-called alternating bond length (ABL) modes, and in particular the longitudinal mode of highest frequency.

Although it seems evident that the interpretation based on vibrational modes is essentially correct, what is still lacking is a systematic study of the behavior of wires with varying numbers of atoms, and surrounded by various lead geometries. Many questions of the basic physics are also still not very well understood: When exactly does the electron-vibration coupling lead to a drop and when an increase in the conductance? Why does there appear to be just a single drop in the experiments of Ref. 18, although the momentum conservation is not exact? What determines the height and width of this drop? Below we aim to discuss the possible answers to some of these questions.

In this paper we concentrate on studying the currentvoltage characteristics of gold wires. We use a SlaterKoster [21] type tight-binding (TB) approach, where the parameters are taken from the non-orthogonal parametrization of Papaconstantopoulos and coworkers 
22, 23, 24, 25]. The use of such a parametrization 26, 27, 28] makes the modeling of atomic wires computationally less intensive as compared with fully ab initio methods. The approach is still microscopic in that it takes into account the symmetries of the atomic $s, p$, and $d$ valence orbitals, which, via hybridization, form the conduction channels. It is also general enough to allow one to model everything within the same framework: we use the parameters to compute the total energy of the wire, and thus optimize the geometry. After this, the normal modes of oscillation and the electron-vibration coupling constants can be computed. Finally, we calculate the transport properties using the non-equilibrium Green function (or Keldysh) approach. Our implementation is very similar to that of Ref. 28 , and the present work is, in essence, an extension of that to inelastic transport. In addition to the full $a b$ initio calculations [19], the effect of electron-vibration interactions on transport through molecular wires has been recently studied by some simple single-level models [29, 30, 31]. The tight-binding approach stands somewhere in between these two extremes.

We compute the conductance to lowest nontrivial order in the electron-vibration coupling constant. There are essentially two well-defined limits which can be studied. In the first limit the vibrational-mode distribution remains in equilibrium due to a strong coupling of the modes to an external equilibrium bath formed by the leads. In the opposite limit the distribution is driven to strong nonequilibrium by the bias voltage. These are the externally damped and the externally undamped limits of Ref. 19. However, in the first limit one should also account for the strong broadening of the vibrational modes. We derive equations which take this into account in a phenomenological manner.

With our simple, self-contained method of optimizing the geometry, we obtain vibrational frequencies which are of the correct order of magnitude, usually to within a factor of two. We study how the positions and heights of the conductance drops due to the electron-vibration coupling vary with elongation of a linear wire, and find a good overall agreement with the experiments of Ref. 18. The height of the conductance steps grows together with the length of the wire, being typically of the order $0.5 \%-5 \%$ of $G_{0}$ for wires of 11 atoms or less. As found in the earlier calculations 19], we find that the highest-frequency longitudinal modes usually couple most strongly, although there seems to be no fundamental reason for a bias toward the "ABL" modes. But in contrast to previous theoretical results, the conductance drop is usually found to occur in two or more consecutive steps which are due to several close-lying longitudinal vibrational modes. Thus we find the "mode selectivity" to be only very approximate. However, the steps can be made to merge into a single one, when we introduce a large enough phenomenological broadening to the vibrational modes, such that the experimentally observed step widths of $\sim 5 \mathrm{meV}$ are accounted for. We also briefly study the conductance signatures of chains which have a

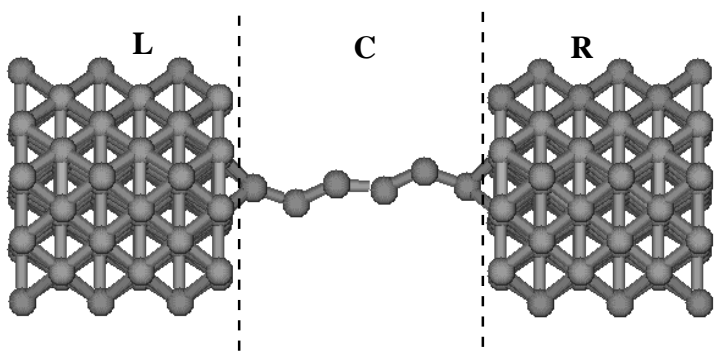

FIG. 1: Geometry A, without "pyramids". A zigzag wire with $N_{c h}=6$ atoms is shown.

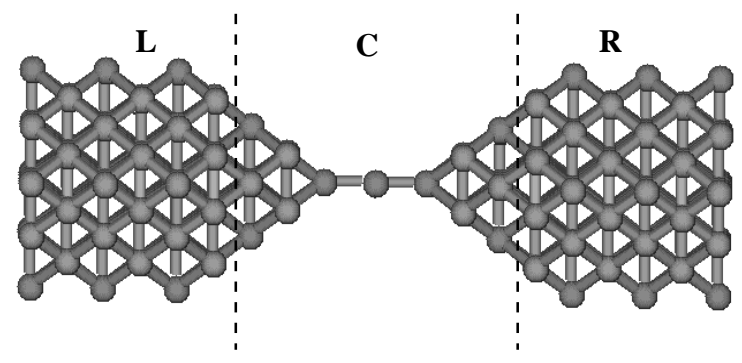

FIG. 2: Geometry B, with "pyramids" and a linear wire of $N_{c h}=3$ atoms.

zigzag-like form, instead of the linear one.

The paper is divided into the following parts. In Sec. III we start by defining the problem, and discussing the electron-vibration coupling. In Sec. III we briefly discuss the calculation of the vibrational modes and the electronvibration coupling constants, as well as our methods of computing the transport. After this, Sec. IV] introduces the important wide-band approximation to the full formalism. In Sec. $\nabla$ we use the formalism to analyze simple tight-binding models, and in Sec. VI the full spd-tightbinding model. Section VII ends with some conclusions and discussion. Technical details which are not of immediate importance are postponed to the appendixes. These include a discussion of the calculation of the matrix elements needed for the coupling constants. Readers mainly interested in the results can directly jump to Secs. VIVII

\section{DEFINITION OF THE PROBLEM}

To model transport through atomic wires, we consider two idealized geometries, shown in Figs. 1] and 2] We call these geometry A and geometry B, respectively. Both involve a gold chain of $N_{c h}$ atoms suspended between two gold leads. As the leads, we simply use semi-infinite "bars", where the repeat unit consists of two layers, with 12 and 13 atoms respectively, mimicking an infinite fcc [001] surface, where the $z$ axis is always chosen parallel to the axis of the wire. The particular choice for the leads should not be very important, as long as they are infinite in one direction, and wider than the contact region. In 
geometry B, the chain connects to small clusters of atoms or "pyramids" on the surfaces (in our case consisting of 9 atoms), making it perhaps the more realistic one of the two. For technical reasons, the geometry is divided into three parts, the semi-infinite left $(L)$ and right $(R)$ leads, and the "central cluster" $(C)$, which also includes the pyramids if any. These parts are indicated in the figures. We shall also consider some simple models where the regions $L, C$, and $R$ are parts of an infinite linear wire.

Our objective is to model the effect of vibrations (or "phonons") of the wire on the transport, when a voltage is applied over the contact. Within a tight-binding picture, the system of electrons coupled to vibrational modes is described by the Hamiltonian

$$
\hat{H}=\hat{H}_{e}+\hat{H}_{v i b}+\hat{H}_{e-v i b},
$$

where

$$
\begin{aligned}
\hat{H}_{e} & =\sum_{i j} d_{i}^{\dagger} H_{i j} d_{j} \\
\hat{H}_{v i b} & =\sum_{\alpha} \hbar \omega_{\alpha} b_{\alpha}^{\dagger} b_{\alpha} \\
\hat{H}_{e-v i b} & =\sum_{i j} \sum_{\alpha} d_{i}^{\dagger} \lambda_{i j}^{\alpha} d_{j}\left(b_{\alpha}^{\dagger}+b_{\alpha}\right) .
\end{aligned}
$$

Here $\omega_{\alpha}$ are the vibrational frequencies, $H_{i j}=\langle i|H| j\rangle$ are the matrix elements of the equilibrium single-electron Hamiltonian $H$ in the atomic-orbital basis $\{|i\rangle\}$, and $\lambda_{i j}^{\alpha}$ are the electron-vibration coupling constants. The index $i$ denotes collectively the atomic sites and orbitals, and $\alpha$ runs from 1 to $3 N_{v i b}$, where $N_{v i b}$ is the number of atoms in the system, which are allowed to vibrate. The creation and annihilation operators for vibrational modes $b_{\alpha}^{\dagger}, b_{\alpha}$ satisfy the bosonic commutation relation $\left[b_{\alpha}, b_{\beta}^{\dagger}\right]=\delta_{\alpha \beta}$. The electronic basis is in general nonorthogonal, with overlap matrix elements $S_{i j}=\langle i \mid j\rangle$. Thus the anticommutator for electron operators $d_{i}^{\dagger}, d_{i}$ is given by $\left\{d_{i}, d_{j}^{\dagger}\right\}=\left[\boldsymbol{S}^{-1}\right]_{i j}$.

Hereafter we denote the matrices with components $A_{i j}$ with a boldface symbol $\boldsymbol{A}$. The matrices $\boldsymbol{H}, \boldsymbol{S}$, and $\boldsymbol{\lambda}^{\alpha}$ are all symmetric in our case. In the spd TB model, the matrix elements $H_{i j}$ and $S_{i j}$ are obtained directly from the parametrization [25]. These can also be used to calculate the the vibrational frequencies $\omega_{\alpha}$ and the coupling constants $\boldsymbol{\lambda}^{\alpha}$, as we shall now describe.

\section{METHODS}

The solution of the inelastic transport problem involves a few rather separated sub-problems: the optimization of the geometry and evaluation of the vibrational modes, estimation of the electron-vibration coupling constants, and finally the calculation of the transport. In the following we give only a brief description of each of them, and refer the reader to appendixes for details. Our basic approach is to solve for the elastic transmission problem exactly, and then to take the electron-vibration coupling into account in a slightly modified version of lowestorder perturbation theory. Other works have considered the so-called self-consistent Born approximation (SCBA) [19, 31], where some of the terms in the perturbation expansions are effectively summed to infinite order. However, this is not essential for describing the basic physics which is involved in the present problem.

\section{A. Vibrational modes and the electron-vibration coupling constants}

The calculation of the vibrational modes requires knowledge of the total (ground-state) energy of the system as a function $E\left(\vec{R}_{k}\right)$ of the ionic coordinates $\vec{R}_{k}$ with $k=1, \ldots, N_{v i b}$. This energy must be minimized to find the equilibrium configuration $\vec{R}_{k}^{(0)}$. Now consider small displacements $\vec{Q}_{k}=\vec{R}_{k}-\vec{R}_{k}^{(0)}$ around the equilibrium. The Hamiltonian describing the oscillations of the ions around $\vec{R}_{k}^{(0)}$ is given by

$$
H_{\text {ion }}=\frac{1}{2} \sum_{k \mu} M_{k} \dot{Q}_{k \mu}^{2}+\frac{1}{2} \sum_{k \mu, l \nu} \mathcal{H}_{k \mu, l \nu} Q_{k \mu} Q_{l \nu},
$$

where $M_{k}$ are the are the ionic masses, $\mu, \nu=x, y, z$ denote Cartesian components of vectors and $\mathcal{H}$ is the Hessian matrix: $\mathcal{H}_{k \mu, l \nu}=\partial^{2} E / \partial R_{k \mu} \partial R_{l \nu}$. This can be diagonalized by the transformation $Q_{k \mu}=\sum_{\alpha=1}^{3 N_{v i b}} A_{k \mu, \alpha} q_{\alpha}$, where $q_{\alpha}$ are the normal coordinates. Thus, we obtain $H_{\text {ion }}=\frac{1}{2} \sum_{\alpha}\left(\dot{q}_{\alpha}^{2}+\omega_{\alpha}^{2} q_{\alpha}^{2}\right)$, where $\omega_{\alpha}\left(\alpha=1, \ldots, 3 N_{v i b}\right)$ are the vibrational frequencies. The transformation matrix $A$ is normalized according to $A^{T} M A=1, M$ being the mass matrix - in our case $M$ is simply a scalar giving the mass of a gold atom. Upon using the canonical quantization prescription $q_{\alpha}=\left(\hbar / 2 \omega_{\alpha}\right)^{1 / 2}\left(b_{\alpha}^{\dagger}+b_{\alpha}\right)$, $\dot{q}_{\alpha}=\mathrm{i}\left(\hbar \omega_{\alpha} / 2\right)^{1 / 2}\left(b_{\alpha}^{\dagger}-b_{\alpha}\right)$, one finally obtains $\hat{H}_{v i b}$ in Eq. (1).

The electron-vibration interaction may be derived as follows [32, 33]. Assume that the electronic singleparticle Hamiltonian $H$ is a function of the ionic coordinates, denoted collectively as $\vec{R}$. Then we may expand $H\left(\vec{R}^{(0)}+\vec{Q}\right) \approx H\left(\vec{R}^{(0)}\right)+\left.\sum_{k} \vec{Q}_{k} \cdot \vec{\nabla}_{k} H\right|_{\vec{Q}=0}$. Defining $\hat{H}_{e}^{\prime}=\sum_{i j} d_{i}^{\dagger}\left\langle i\left|H\left(\vec{R}^{(0)}+\vec{Q}\right)\right| j\right\rangle d_{j}$, inserting the expansion, and using the canonical quantization for $q_{\alpha}$ again, one finds $\hat{H}_{e}^{\prime}=\hat{H}_{e}+\hat{H}_{e-v i b}$ [cf. Eq. (1)], where $H \equiv H\left(\vec{R}^{(0)}\right)$, and the electron-vibration coupling constants are given by

$$
\lambda_{i j}^{\alpha}=\lambda_{0}\left(\frac{\hbar}{2 \omega_{\alpha}}\right)^{1 / 2} \sum_{k \mu} M_{i j}^{k \mu} A_{k \mu, \alpha},
$$

where $M_{i j}^{k \mu}=\left\langle i\left|\nabla_{k \mu} H\right|_{\vec{Q}=0} \mid j\right\rangle$. The calculation of these matrix elements is explained in Appendix A In Eq. (2) we have added a dimensionless factor $\lambda_{0}$ to describe the strength of the coupling - in the physical case $\lambda_{0}=1$. 


\section{B. Propagator formalism}

Use of a local basis allows one to partition the electronic Hamiltonian and overlap matrices into parts according to the division in $L, C$ and $R$ regions:

$$
\boldsymbol{H}=\left[\begin{array}{lll}
\boldsymbol{H}_{L L} & \boldsymbol{H}_{L C} & \boldsymbol{H}_{L R} \\
\boldsymbol{H}_{C L} & \boldsymbol{H}_{C C} & \boldsymbol{H}_{C R} \\
\boldsymbol{H}_{R L} & \boldsymbol{H}_{R C} & \boldsymbol{H}_{R R}
\end{array}\right], \boldsymbol{S}=\left[\begin{array}{lll}
\boldsymbol{S}_{L L} & \boldsymbol{S}_{L C} & \boldsymbol{S}_{L R} \\
\boldsymbol{S}_{C L} & \boldsymbol{S}_{C C} & \boldsymbol{S}_{C R} \\
\boldsymbol{S}_{R L} & \boldsymbol{S}_{R C} & \boldsymbol{S}_{R R}
\end{array}\right] .
$$

Although the dimension of the problem is infinite, its single-particle nature allows for very effective methods of solution, as long as we may assume that $\boldsymbol{H}_{R L}=\boldsymbol{H}_{L R}^{T} \approx$ 0 and $\boldsymbol{S}_{R L}=\boldsymbol{S}_{L R}^{T} \approx 0$, which we shall do. We shall use the method of non-equilibrium Green functions (NEGF). In this method, one can restrict the problem only to the $C$ part by introducing energy-dependent lead self-energies which take into account the presence of the semi-infinite $L$ and $R$ leads in an exact way.

The quantity from which all elastic transport properties may be extracted, is the retarded Green function of the $C$ part in the absence of electron-vibration coupling. We call it $\tilde{\boldsymbol{G}}^{r}$, and it may be written as $\tilde{\boldsymbol{G}}^{r}(\epsilon)=$ $\left[\epsilon \boldsymbol{S}_{C C}-\boldsymbol{H}_{C C}-\boldsymbol{\Sigma}_{L}^{r}-\boldsymbol{\Sigma}_{R}^{r}\right]^{-1}$. The lead self-energy $\boldsymbol{\Sigma}_{L}^{r}$ is given by $\boldsymbol{\Sigma}_{L}^{r}=\boldsymbol{t}_{C L} \boldsymbol{g}_{L L}^{r} \boldsymbol{t}_{L C}$, and $\boldsymbol{\Gamma}_{L}=\mathrm{i}\left(\boldsymbol{\Sigma}_{L}^{r}-\boldsymbol{\Sigma}_{L}^{a}\right)$, where we define $\boldsymbol{t}_{C L}=\boldsymbol{H}_{C L}-\epsilon \boldsymbol{S}_{C L}$. The matrix $\boldsymbol{g}_{L L}^{r}(\epsilon)=\left[\left(\epsilon+\mathrm{i} \gamma_{L} / 2\right) \boldsymbol{S}_{L L}-\boldsymbol{H}_{L L}\right]^{-1}$ is the lead (surface) Green function, where $\gamma_{L}=0^{+}$. Similar equations hold for $\boldsymbol{\Sigma}_{R}^{r}$. The lead Green functions $\boldsymbol{g}_{L L}^{r}$ and $\boldsymbol{g}_{R R}^{r}$ are "surface" Green functions for the semi-infinite leads. We compute these with the so-called decimation technique [34], using TB parameters for bulk 28] in the case of the full spd model. The electron-vibration interaction gives rise to further self-energies, as will be discussed below.

The vibrational modes should in principle be treated in an analogous way, by introducing lead self-energies for their propagators. However, here we restrict the modes strictly to the wire of $N_{c h}$ atoms within the $C$ region (i.e. $\left.N_{v i b}=N_{c h}\right)$ and use the corresponding normal-mode basis for them. Thus the number of modes which we have to consider is only $3 N_{c h}$, and their "lead coupling" is taken into account only in a phenomenological way.

More details on the propagator technique, including the expressions for the phonon propagators and all selfenergy diagrams, are given in Appendix C]

\section{Calculation of current}

The most important physical observable which we are interested in is the electric (charge) current through the atomic wire, when a voltage $V$ is applied. We denote $e V=\mu_{L}-\mu_{R}$, where $\mu_{L, R}$ are the $L$ and $R$ side chemical potentials, and $e>0$ is the absolute value of electron charge. We also define $f_{L, R}(\epsilon)=f\left(\epsilon-\mu_{L, R}\right)$, where $f(\epsilon)=1 /[\exp (\beta \epsilon)+1]$ is the Fermi function, and $\beta=$ $1 / k_{B} T$ is the inverse temperature.
It may be shown that the current flowing through the interface from $L$ to $C(C$ to $R)$ in stationary state is given by (see Appendix B]

$$
I^{\Omega}= \pm \frac{2 e}{\hbar} \int \frac{\mathrm{d} \epsilon}{2 \pi} \operatorname{Tr}\left[\boldsymbol{G}_{C \Omega}^{+-}(\epsilon) \boldsymbol{t}_{\Omega C}(\epsilon)-\boldsymbol{t}_{C \Omega}(\epsilon) \boldsymbol{G}_{\Omega C}^{+-}(\epsilon)\right]
$$

where $\Omega=L(R)$ is chosen with the upper (lower) sign, and the factor 2 accounts for spin degeneracy. The Green functions $\boldsymbol{G}^{+-}$are defined in Appendix Cl by Eq. (C1). Developing Eq. (3) further, it is convenient to split it into two parts: $I^{L, R}=I_{e l}+I_{i n e l}^{L, R}$, where

$$
\begin{aligned}
& I_{e l}=\frac{2 e}{\hbar} \int \frac{\mathrm{d} \epsilon}{2 \pi} \operatorname{Tr}\left[\boldsymbol{G}^{r} \boldsymbol{\Gamma}_{R} \boldsymbol{G}^{a} \boldsymbol{\Gamma}_{L}\right]\left(f_{L}-f_{R}\right) \\
& I_{\text {inel }}^{L, R}= \pm \frac{2 e}{\hbar} \mathrm{i} \int \frac{\mathrm{d} \epsilon}{2 \pi} \operatorname{Tr}\left\{\boldsymbol{G}^{a} \boldsymbol{\Gamma}_{L, R} \boldsymbol{G}^{r}\right. \\
&\left.\times\left[\left(f_{L, R}-1\right) \boldsymbol{\Sigma}_{e-v i b}^{+-}-f_{L, R} \boldsymbol{\Sigma}_{e-v i b}^{-+}\right]\right\} .
\end{aligned}
$$

Here we define the full retarded and advanced Green functions $\boldsymbol{G}^{r, a}$, where $\boldsymbol{G}^{r}=\left[\epsilon \boldsymbol{S}_{C C}-\boldsymbol{H}_{C C}-\boldsymbol{\Sigma}_{L}^{r}-\boldsymbol{\Sigma}_{R}^{r}-\right.$ $\left.\boldsymbol{\Sigma}_{e-v i b}^{r}\right]^{-1}$ and $\boldsymbol{G}^{a}=\left[\boldsymbol{G}^{r}\right]^{\dagger}$. The new self-energies $\boldsymbol{\Sigma}_{e-v i b}^{r}$ and $\boldsymbol{\Sigma}_{e-v i b}^{ \pm \mp}$ are due to the electron-vibration interaction, and they are discussed in more detail in Appendix $C$ Since they vanish in the absence of $\lambda^{\alpha}$, we call the $I_{\text {inel }}^{L, R}$ part an "inelastic" current, while $I_{e l}$ is the "elastic" part 31].

If we do lowest-order perturbation theory with respect to $\boldsymbol{\lambda}^{\alpha}$, we may expand $\boldsymbol{G}^{r}=\tilde{\boldsymbol{G}}^{r}+\tilde{\boldsymbol{G}}^{r} \boldsymbol{\Sigma}_{e-v i b}^{r} \tilde{\boldsymbol{G}}^{r}+\cdots$. In this way the elastic current is split into two parts as $I_{e l}=I_{e l}^{0}+\delta I_{e l}$, where $\delta I_{e l}$ is an "elastic correction". We find

$$
\begin{aligned}
& I_{e l}^{0}=\frac{2 e}{\hbar} \int \frac{\mathrm{d} \epsilon}{2 \pi} \operatorname{Tr}\left[\tilde{\boldsymbol{G}}^{r} \boldsymbol{\Gamma}_{R} \tilde{\boldsymbol{G}}^{a} \boldsymbol{\Gamma}_{L}\right]\left(f_{L}-f_{R}\right) \\
& \delta I_{e l}=\frac{4 e}{\hbar} \int \frac{\mathrm{d} \epsilon}{2 \pi} \operatorname{Re} \operatorname{Tr}\left[\boldsymbol{\Gamma}_{L} \tilde{\boldsymbol{G}}^{r} \boldsymbol{\Sigma}_{e-v i b}^{r} \tilde{\boldsymbol{G}}^{r} \boldsymbol{\Gamma}_{R} \tilde{\boldsymbol{G}}^{a}\right] \\
& \times\left(f_{L}-f_{R}\right) \\
& I_{\text {inel }}^{L, R}= \pm \frac{2 e}{\hbar} \mathrm{i} \int \frac{\mathrm{d} \epsilon}{2 \pi} \operatorname{Tr}\left\{\tilde{\boldsymbol{G}}^{a} \boldsymbol{\Gamma}_{L, R} \tilde{\boldsymbol{G}}^{r}\right. \\
&\left.\times\left[\left(f_{L, R}-1\right) \boldsymbol{\Sigma}_{e-v i b}^{+-}-f_{L, R} \boldsymbol{\Sigma}_{e-v i b}^{-+}\right]\right\}
\end{aligned}
$$

A proof of current conservation, that is $I^{L}=I^{R} \equiv I$, is sketched in Appendix D.

Besides the charge current, other interesting observables would be the heat current (or power dissipation) [19], current noise [35], and possibly spin current in case of magnetic materials. We only consider the charge current here, as it is the only one which can easily be measured. More specifically, we shall be interested in the differential conductance $G(V)=\mathrm{d} I / \mathrm{d} V$ and its derivative, since these quantities reveal the signatures of the vibrational-mode coupling most clearly. 


\section{WIDE-BAND LIMIT}

Even in the case of the perturbative current formulas [Eqs. [5] ], the expressions will involve double energy integrals which can be very cumbersome to evaluate. These general formulas are discussed more in Appendix E where they are rewritten in terms of distribution functions and energy-dependent transport coefficients. However, the existence of different energy scales in the problem allows us to make an important simplification.

The energies of the vibrational modes are on the order of $10 \mathrm{meV}$, so that we are only interested in the differential conductance for voltages up to $V \approx 40 \mathrm{mV}$, at most. Together with the temperature $T \approx 4.2 \mathrm{~K}$, this determines the width of the energy window around the Fermi energy $\left(\epsilon_{F}\right)$ which is important for transport. However, for the atomic wires which we are considering, the electronic density of states tends to vary at the much larger energy scales $\sim 1 \mathrm{eV}$ around $\epsilon_{F}$. Thus, to a good approximation, we may neglect this energy dependence, and simply evaluate all the electronic Green functions at the Fermi energy. This approximation is often called the "wide-band limit" (WBL).

\section{A. Current}

In the WBL approximation, the current expressions of Appendix E may simplified considerably, since some of the energy integrals may be done analytically. In this case the current $I$ is easily divided into symmetric and asymmetric parts, according to the symmetry of their contributions to $G(V)$ under the reversal of $V$ [36]. Thus $I=I^{\text {sym }}+I^{a s y}$, where the symmetric current is

$$
\begin{aligned}
& I^{s y m}=\frac{2 e^{2}}{h} T_{0} V+\frac{2 e}{h} \sum_{\alpha} \int_{0}^{\infty} \mathrm{d} \omega_{1} \rho_{\alpha}\left(\omega_{1}\right)\{ \\
& {\left[\left(T_{\alpha}^{e c}+T_{\alpha}^{i n}\right)\left(2 N_{\alpha}\left(\omega_{1}\right)+1\right)-\left(T_{\alpha}^{e c L R}+T_{\alpha}^{i n}\right)\right] e V} \\
& \left.+\left(T_{\alpha}^{e c L R}+T_{\alpha}^{i n}\right)\left(\frac{\omega_{1}-e V}{e^{\beta\left(\omega_{1}-e V\right)}-1}-\frac{\omega_{1}+e V}{e^{\beta\left(\omega_{1}+e V\right)}-1}\right)\right\}
\end{aligned}
$$

and the asymmetric part is

$$
\begin{aligned}
& I^{a s y}=-\frac{2 e}{h} \sum_{\alpha} \int_{-\infty}^{\infty} \mathrm{d} \omega_{1} \frac{1}{2 \pi} \operatorname{Re}\left[d_{\alpha}^{r}\left(\omega_{1}\right)\right] T_{\alpha}^{a s y} \\
& \quad \times\left[2 n\left(\omega_{1}\right) \omega_{1}-\frac{\omega_{1}-e V}{e^{\beta\left(\omega_{1}-e V\right)}-1}-\frac{\omega_{1}+e V}{e^{\beta\left(\omega_{1}+e V\right)}-1}\right] .
\end{aligned}
$$

Here $n(\epsilon)=1 /[\exp (\beta \epsilon)-1]$ is the Bose distribution and $N_{\alpha}$ is the voltage-dependent mode distribution, to be discussed shortly. The function $\rho_{\alpha}$ is the vibrational density of states (DOS), given in general by Eq. (C12). We approximate it here by using the imaginary part of $d_{\alpha}^{r}$ in
Eq. (C8), that is

$$
\rho_{\alpha}(\epsilon)=\frac{1}{\pi} \frac{\eta / 2}{\left(\epsilon-\hbar \omega_{\alpha}\right)^{2}+\eta^{2} / 4}-\frac{1}{\pi} \frac{\eta / 2}{\left(\epsilon+\hbar \omega_{\alpha}\right)^{2}+\eta^{2} / 4},
$$

where we take $\eta$ as a finite phenomenological parameter describing the effect of coupling the vibrational modes to an external bath. This bath is provided by the leads [31]. However, we are neglecting any renormalizations of the bare frequencies $\omega_{\alpha}$, so that the main purpose of $\eta$ here is to broaden the DOS. Similarly, $\operatorname{Re} d_{\alpha}^{r}$ is obtained from Eq. (C8).

The current involves a number of transport coefficients, which determine the shape of the current-voltage characteristics. The coefficients of $I^{\text {sym }}$ may be computed as follows:

$$
\begin{gathered}
T_{0}=\left.\operatorname{Tr}\left[\tilde{\boldsymbol{G}}^{r} \boldsymbol{\Gamma}_{R} \tilde{\boldsymbol{G}}^{a} \boldsymbol{\Gamma}_{L}\right]\right|_{\epsilon_{F}}, \\
T_{\alpha}^{i n}=\left.\operatorname{Tr}\left[\tilde{\boldsymbol{G}}^{r} \boldsymbol{\Gamma}_{R} \tilde{\boldsymbol{G}}^{a} \boldsymbol{\lambda}^{\alpha} \tilde{\boldsymbol{G}}^{a} \boldsymbol{\Gamma}_{L} \tilde{\boldsymbol{G}}^{r} \boldsymbol{\lambda}^{\alpha}\right]\right|_{\epsilon_{F}},
\end{gathered}
$$

$$
T_{\alpha}^{e c}=\left.2 \operatorname{Re} \operatorname{Tr}\left[\tilde{\boldsymbol{G}}^{r} \boldsymbol{\Gamma}_{R} \tilde{\boldsymbol{G}}^{a} \boldsymbol{\Gamma}_{L} \tilde{\boldsymbol{G}}^{r} \boldsymbol{\lambda}^{\alpha} \tilde{\boldsymbol{G}}^{r} \boldsymbol{\lambda}^{\alpha}\right]\right|_{\epsilon_{F}},
$$

$$
\begin{aligned}
T_{\alpha}^{e c L R} & =T_{\alpha}^{e c L}+T_{\alpha}^{e c R} \\
& =\left.\operatorname{Re} \operatorname{Tr}\left[\tilde{\boldsymbol{G}}^{r} \boldsymbol{\Gamma}_{R} \tilde{\boldsymbol{G}}^{a} \boldsymbol{\Gamma}_{L} \tilde{\boldsymbol{G}}^{r} \boldsymbol{\lambda}^{\alpha}\left(\tilde{\boldsymbol{G}}^{r}-\tilde{\boldsymbol{G}}^{a}\right) \boldsymbol{\lambda}^{\alpha}\right]\right|_{\epsilon_{F}}
\end{aligned}
$$

Here $T_{\alpha}^{e c L R}$ is a sum of the two coefficients $T_{\alpha}^{e c L}$ and $T_{\alpha}^{e c R}$ defined in Eqs. (E6). The coefficient $T_{\alpha}^{i n}$ is always positive, while $T_{\alpha}^{e c L R}$ and $T_{\alpha}^{e c}$ can apparently have either sign. The latter two represent interferences between various processes, and are responsible for the enhanced backscattering needed for the conductance drops. The asymmetric coefficient

$$
T_{\alpha}^{a s y}=\left.\operatorname{Re} \operatorname{Tr}\left[\tilde{\boldsymbol{G}}^{r} \boldsymbol{\Gamma}_{R} \tilde{\boldsymbol{G}}^{a} \boldsymbol{\Gamma}_{L} \tilde{\boldsymbol{G}}^{r} \boldsymbol{\lambda}^{\alpha} \tilde{\boldsymbol{G}}^{r}\left(\boldsymbol{\Gamma}_{R}-\boldsymbol{\Gamma}_{L}\right) \boldsymbol{G}^{a} \boldsymbol{\lambda}^{\alpha}\right]\right|_{\epsilon_{F}}
$$

vanishes for sufficiently symmetric junctions, making $I^{a s y}=0$. For us, this is always the case, even with zigzag chains.

\section{B. Mode distribution}

Our approximation is essentially that of lowest-order perturbation theory in the coupling constant $\boldsymbol{\lambda}^{\alpha}$. However, as explained in Appendix [C] there is a natural way of extending the theory somewhat further by taking into account the "phonon polarizations" in a voltagedependent distribution function $N_{\alpha}$. This is given by Eq. (C14), i.e.

$$
N_{\alpha}(\epsilon)=-\frac{1}{2} \frac{\operatorname{Im} \Pi_{\alpha}^{+-}(\epsilon)+n(\epsilon) \eta \epsilon / \hbar \omega_{\alpha}}{\operatorname{Im} \Pi_{\alpha}^{r}(\epsilon)-\eta \epsilon / 2 \hbar \omega_{\alpha}},
$$


where $\eta$ is the same bath-coupling parameter as in $\rho_{\alpha}$. Here $\operatorname{Im} \Pi_{\alpha}^{+-}$and $\operatorname{Im} \Pi_{\alpha}^{r}$ are imaginary parts of the phonon polarizations (see Appendix C). In the WBL one may show that

$$
\begin{aligned}
2 \pi & \operatorname{Im} \Pi_{\alpha}^{+-}(\epsilon) \\
\approx & \left.\operatorname{Tr}\left[\boldsymbol{\lambda}^{\alpha} \tilde{\boldsymbol{G}}^{r} \boldsymbol{\Gamma}_{L} \tilde{\boldsymbol{G}}^{a} \boldsymbol{\lambda}^{\alpha} \tilde{\boldsymbol{G}}^{r} \boldsymbol{\Gamma}_{R} \tilde{\boldsymbol{G}}^{a}\right]\right|_{\epsilon_{F}} \\
& \times\left[\frac{\epsilon+e V}{e^{\beta(\epsilon+e V)}-1}+\frac{\epsilon-e V}{e^{\beta(\epsilon-e V)}-1}\right] \\
+ & \left\{\left.\operatorname{Tr}\left[\boldsymbol{\lambda}^{\alpha} \tilde{\boldsymbol{G}}^{r} \boldsymbol{\Gamma}_{L} \tilde{\boldsymbol{G}}^{a} \boldsymbol{\lambda}^{\alpha} \tilde{\boldsymbol{G}}^{r} \boldsymbol{\Gamma}_{L} \tilde{\boldsymbol{G}}^{a}\right]\right|_{\epsilon_{F}}\right. \\
& \left.+\left.\operatorname{Tr}\left[\boldsymbol{\lambda}^{\alpha} \tilde{\boldsymbol{G}}^{r} \boldsymbol{\Gamma}_{R} \tilde{\boldsymbol{G}}^{a} \boldsymbol{\lambda}^{\alpha} \tilde{\boldsymbol{G}}^{r} \boldsymbol{\Gamma}_{R} \tilde{\boldsymbol{G}}^{a}\right]\right|_{\epsilon_{F}}\right\} \frac{\epsilon}{e^{\beta \epsilon}-1}
\end{aligned}
$$

and

$$
\operatorname{Im} \Pi_{\alpha}^{r}(\epsilon) \approx-(\epsilon / \pi) \operatorname{Tr}\left[\boldsymbol{\lambda}^{\boldsymbol{\alpha}} \operatorname{Im} \tilde{\boldsymbol{G}}^{r} \boldsymbol{\lambda}^{\boldsymbol{\alpha}} \operatorname{Im} \tilde{\boldsymbol{G}}^{r}\right],
$$

which is proportional to the electron-hole damping rate of Ref. 36. Using $\tilde{\boldsymbol{G}}^{r}\left(\boldsymbol{\Gamma}_{L}+\boldsymbol{\Gamma}_{R}\right) \tilde{\boldsymbol{G}}^{a}=-2 \operatorname{Im} \tilde{\boldsymbol{G}}^{r}$, one may easily show that for $V=0$ Eqs. (14)-(16) indeed yield $N_{\alpha}(\epsilon)=n(\epsilon)$ for any $\eta$.

Here it is important to note a few things. In the expression for the distribution function, the limit $\eta \rightarrow 0+$ corresponds to the case where the vibrational modes are uncoupled from leads. Supposing that one also wishes to take the phonon polarizations to zero, which is formally accomplished by taking $\lambda_{0} \rightarrow 0$, one discovers an interesting thing: the two limits do not commute.

If we take first the limit $\eta \rightarrow 0$, then the result actually becomes independent of $\lambda_{0}$, since $\Pi_{\alpha}^{r}, \Pi_{\alpha}^{+-} \propto \lambda_{0}^{2}$ and the $\lambda_{0}^{2}$-factors cancel. A physical interpretation can be described as follows. If the vibrational modes are not coupled to any external bath, then even an infinitesimally small coupling constant can eventually lead to a stationary state with a strongly nonequilibrium mode occupation. Here emission and absorption of phonons are in balance, and hence there is no net energy transfer between the electrons and the vibrations. Following Ref. 19, we call this the externally undamped limit, although our way of computing $N_{\alpha}$ is quite different. In this case the voltage-dependence of $N_{\alpha}\left(\hbar \omega_{\alpha}\right)$ shows a sharp kink at $V=\hbar \omega_{\alpha} / e$, and a subsequent linear increase [19].

In the opposite case, where $\lambda_{0} \rightarrow 0$ first, the expression becomes independent of $\eta$, and we recover the Bose distribution. This corresponds to the limit where the vibrational modes are strongly damped by coupling to a heat bath which is in equilibrium. This is the externally damped limit. However, for a finite $\boldsymbol{\lambda}^{\alpha}$ this limit can only be reached with a large enough finite $\eta$. Thus the externally damped limit should also imply a considerable broadening of the vibrational modes.

Note that in both of the above limits, $N_{\alpha}$ is zeroth order in $\lambda_{0}$. In these two cases our expression for $I^{\text {sym }}$ [Eq. [6)] is indeed of second order in $\lambda_{0}$, and there should be no corrections within that order. In general, however, Eq. (14) for $N_{\alpha}$ generates terms of all orders in $\lambda_{0}^{2}$. These are not, strictly speaking, warranted, because they represent only a small subset of all possible higher-order terms in the current.

\section{Further discussion}

Apart from the $\rho_{\alpha}$-weighted integral, the only difference between Eq. (66) and the approximation of Ref. 36 for $I^{s y m}$ is that $T_{\alpha}^{e c} \neq T_{\alpha}^{e c L R}$. The difference $T_{\alpha}^{e c}-T_{\alpha}^{e c L R}$ is proportional to $\operatorname{Re} \tilde{\boldsymbol{G}}^{r}$, and is typically very small. It could well be neglected. If $N_{\alpha} \equiv$ constant, then the direction and size of the conductance step is solely determined by the combination $T_{\alpha}^{e c L R}+T_{\alpha}^{i n}$. Since $T_{\alpha}^{i n}$ is positive, the $I_{\text {inel }}$ part of the current always tends to increase the conductance. This can be seen as a result of the appearance a new, inelastic conduction "channel" when $\mathrm{eV}>\hbar \omega_{\alpha}$. However, numerically one finds that the coefficient $T_{\alpha}^{e c L R}$, due to interferences between various elastic processes, is typically negative and $\left|T_{\alpha}^{e c L R}\right|>T_{\alpha}^{i n}$ when $T_{0} \approx 1$. Thus, the net effect of the vibrational coupling is a decrease in the conductance. In general, the voltage-dependence of $N_{\alpha}$ also affects the shape of $G(V)$. Since $T_{\alpha}^{e c}+T_{\alpha}^{i n}$ is usually also negative, the effect of local heating is to give a finite negative slope to $G(V)$ after the step. There can also be an increase in the apparent height of the step.

By using the vibrational DOS of Eq. (8), we are neglecting broadenings and frequency shifts due to the electron-vibration coupling. The shifts are given by the quantities $\operatorname{Re} \Pi_{\alpha}^{r}$ and, using Eq. (C10), we estimate them to be on the order of $-1 \mathrm{meV}$. Neglecting the broadenings is certainly justified, since they are typically on the order of $\left|\operatorname{Im} \Pi_{\alpha}^{r}\left(\hbar \omega_{\alpha}\right)\right| / \hbar \omega_{\alpha} \lesssim 10^{-2}$, which is much smaller than the usual $k_{B} T / \hbar \omega_{\alpha}$ due to temperatures $T \sim 1 \mathrm{~K}[19]$. If we assume also $\eta$ to be small compared to the other relevant energy scales, i.e. if $\left|\operatorname{Im} \Pi_{\alpha}^{r}\right|, \eta \ll k_{B} T \ll \hbar \omega_{\alpha}$, then $\rho_{\alpha}\left(\omega_{1}\right) \approx \delta\left(\omega_{1}-\hbar \omega_{\alpha}\right)-\delta\left(\omega_{1}+\hbar \omega_{\alpha}\right)$, and the $\omega_{1}$ integral in Eq. (6) may be done analytically. The calculation of the lead-coupling effects is difficult, but it is very possible that they can lead to broadenings $\eta / \hbar \omega_{\alpha} \sim 1$. Thus, the validity of the delta-function approximation of $\rho_{\alpha}$ may be questioned. Naturally, the lead coupling can also shift the vibrational frequencies.

Note also that $N_{\alpha}(\epsilon)$ diverges if $\beta \epsilon \rightarrow 0$. Since in Eq. (66) $N_{\alpha}(\epsilon)$ is evaluated at $\epsilon \sim \hbar \omega_{\alpha}$, there can be an arbitrarily large renormalization of the zero-bias conductance if there are very low-frequency vibrational modes $\left(\hbar \omega_{\alpha} \sim k_{B} T\right)$ with a strong coupling to the electrons. In such a case, the theory appears to break down. With linear wires the condition $k_{B} T \ll \hbar \omega_{\alpha}$ is easily satisfied for all strongly coupled modes $\alpha$, but in case of the zigzag-chains (and/or other materials) it may not be. In any case, we limit our study mostly to the linear wires in this paper. We note that these restrictions are present also in Ref. [36], where $\eta=0^{+}$. In fact, in our formulation the problem is perhaps partly corrected by the presence of the "broadening factor" $\rho_{\alpha}$. In general 


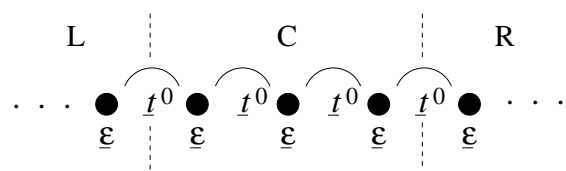

FIG. 3: Infinite multi-orbital nearest-neighbor chain in equilibrium, where the sites are separated by a distance $a$.

$\int_{0}^{\infty} \mathrm{d} \omega_{1} \rho_{\alpha}\left(\omega_{1}\right)<1$, and when $\hbar \omega_{\alpha} \rightarrow 0$, the whole function tends to zero. Thus low-frequency modes contribute to the current with a very small weight.

\section{SIMPLE CHAIN MODELS}

Here we shall first present some example results, using an adaptation of the simple chain models of Refs. 37 and 20. Only after this we turn to the full spd-tight binding parametrization. Chain models of this kind are very appealing, because they can be studied analytically to a large extent, and allow us to make our main points in a simple fashion.

The single-particle Hamiltonians $\boldsymbol{H}$ which we consider, are of the block-tridiagonal form

$$
\boldsymbol{H}=\left[\begin{array}{ccccc}
\ddots & \ddots & \ddots & & \\
& \underline{t}_{i, i-1} & \underline{\epsilon} & \underline{t}_{i, i+1} & \\
& & \ddots & \ddots & \ddots
\end{array}\right],
$$

graphically depicted in Fig. 3. Here $\underline{\epsilon}$ includes the onsite energies, and $\underline{t}_{i, i+1}$ are the inter-site hopping matrices between sites $i$ and $i+1$. They are modulated as a function of the longitudinal atomic displacements $Q_{i}$ as $\underline{t}_{i, i+1}(Q)=\underline{t}^{0}+\underline{t}^{\prime}\left(Q_{i}-Q_{i+1}\right)$. We only consider orthogonal tight-binding here, such that $\boldsymbol{S}=\mathbf{1}$. The chain is split into three parts, where the $C$ part has $N_{c h}$ atoms. The $L$ and $R$ "leads" are semi-infinite chains. The displacements $Q_{i}$ are restricted to the $C$ part, where the Hessian is assumed to be of the form

$$
\mathcal{H}=K\left[\begin{array}{ccccc}
\ddots & \ddots & \ddots & & \\
& -1 & 2 & -1 & \\
& & \ddots & \ddots & \ddots
\end{array}\right],
$$

with fixed boundary conditions at the ends [20]. Unlike in the spd model to be explained below, here the spring constant $K$ is taken as a new, separate parameter. The vibrational modes are simply obtained by diagonalizing this $\mathcal{H}$.

\section{A. Single $(s)$ orbital: discussion}

To remind ourselves of some of the well-known arguments, let us first discuss the simple single-orbital orthogonal model of Ref. 20].
If we assume that charge neutrality is achieved by one electron per atomic site, then the Fermi energy $\epsilon_{F}$ lies exactly in the middle of the band $-2\left|t_{0}\right|<\epsilon-\epsilon_{0}<2\left|t_{0}\right|$, where $\epsilon_{0}$ is the on-site energy, and $t_{0}$ is the inter-site hopping. The Fermi wavevector is then $k_{F}=\pi / 2 a$, where $a$ is the inter-atomic distance. Since the chain has full translational invariance, momentum conservation dictates that only vibrational modes with the wave vector $q_{v i b}=2 k_{F}=\pi / a$ may be excited, via the backscattering of Fermi-point electrons. Furthermore, if the atomic orbitals are invariant with respect to rotations around the axis of the chain ( $s$-wave, say) the modes can only be longitudinal ones. As it happens, $q_{v i b}=\pi / a$ corresponds exactly to the Brillouin zone boundary and thus to the highest-frequency modes.

More technically, the momentum conservation can be seen to follow from the form of the Fourier-transforms $M_{k_{1}, k_{2}}^{q} \propto \delta_{k_{1}-k_{2}, q}$ of the coupling matrix elements $M_{i j}^{k}$ 32]. We note that the momentum conservation can only be strictly valid if one considers the actual vibrational modes of the whole infinite chain. Interestingly, it appears to remain approximately valid even if the vibrational modes are restricted only to a small, finite part of the chain [20]. Indeed, as found in Ref. 20, when the Fermi energy is in the middle of the band, there is only a single visible step in the conductance. But, as we shall discuss next, this appears to be somewhat accidental.

\section{B. Two $\left(s\right.$ and $\left.p_{z}\right)$ orbitals}

Although such a single-orbital model can correctly describe the most important experimental observations, it neglects some details of realistic gold wires. Although atomic gold contacts and chains typically have only one fully open conduction channel at the Fermi surface, this channel is actually formed from the hybridization on multiple orbitals with rotational symmetry around the chain axis $\left(s, p_{z}, d_{3 z^{2}-r^{2}}\right)$. Thus we consider here a simple generalization of the above chain model to the case of two orbitals, with $s$ and $p_{z}$ characters, respectively. In Table 【 we show a set of example parameters for this model. The resulting transmission and DOS curves are shown in Fig. 4 and the conductance-voltage characteristics in Fig. 5. The density of states is defined as $\mathcal{D}_{i}(\epsilon)=-(1 / \pi) \sum_{\alpha} \operatorname{Im} G_{i \alpha, i \alpha}(\epsilon)$, where $i$ now stands for atomic sites and $\alpha$ for orbitals.

The results are very similar to those of the singleorbital chain [20], but the situation is a bit closer to what happens in the full spd model. For example, the hybridization of $s$ and $p_{z}$ result in a gap in the DOS. If we still populate the chain with one electron per site, the Fermi energy will again be in the middle of the lower band (the " $s$ band"), and there is only a single drop in the conductance. This is illustrated by the $\epsilon_{F}=-0.4 \mathrm{eV}$ case in Fig. 5] But for $\epsilon_{F}$ 's deviating from the center of the band, we find that the conductance step generally consists of several substeps, although the total step height 


\begin{tabular}{lll}
\hline Quantity & Symbol & Value \\
\hline \hline Number of chain atoms & $N_{c h}$ & 6 \\
$s$-orbital energy & $\epsilon_{s s}$ & $0.0 \mathrm{eV}$ \\
$p$-orbital energy & $\epsilon_{p p}$ & $1.0 \mathrm{eV}$ \\
Bare $s-s$ hopping & $t_{s s}^{0}$ & $-0.5 \mathrm{eV}$ \\
Bare $p-p$ hopping & $t_{p p}^{0}$ & $0.3 \mathrm{eV}$ \\
Bare $s-p$ hopping & $t_{s p}^{0}=-t_{p s}^{0} 0.35 \mathrm{eV}$ \\
$s-s$ hopping modulation & $t_{s s}^{\prime}$ & $-0.3 \mathrm{eV}$ \\
$p-p$ hopping modulation & $t_{p p}^{\prime}$ & $0.3 \mathrm{eV}$ \\
$s-p$ hopping modulation & $t_{s p}^{\prime}=-t_{p s}^{\prime} 0.3 \mathrm{eV}$ \\
Fermi energy & $\epsilon_{F}$ & $-0.4-0.2 \mathrm{eV}$ \\
Atomic Mass & $M$ & $197 \mathrm{a} . \mathrm{m} . \mathrm{u}$. \\
Spring constant & $K$ & $2.0 \mathrm{eV} / \AA^{2}$ \\
Temperature & $T$ & $1.0-4.2 \mathrm{~K}$ \\
Phonon broadening & $\eta$ & $0.002 \mathrm{meV}$ \\
\hline
\end{tabular}

TABLE I: Parameters for the $s p_{z}$ schain. Here $t_{i j}^{0}$ are elements of the matrix $\underline{t}^{0}$, for example.

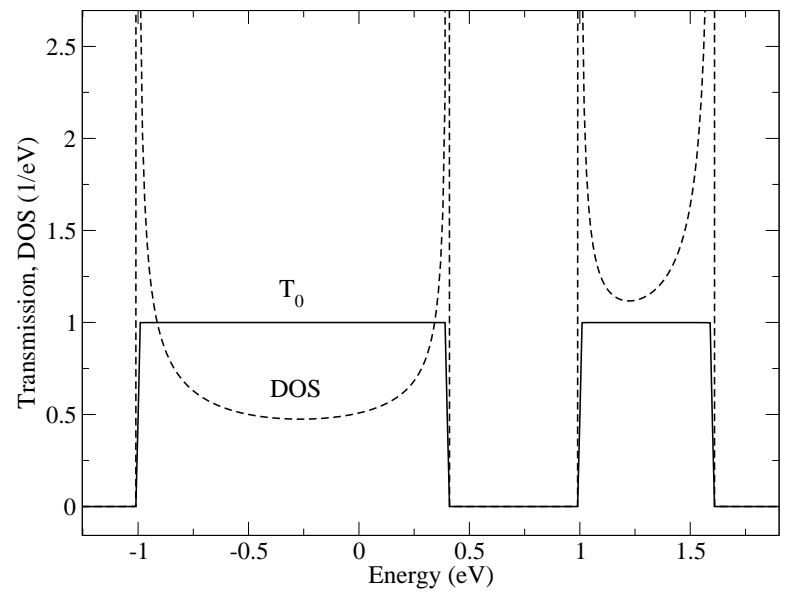

FIG. 4: Elastic transmission $T_{0}$ (solid line) and density of states (dashed line) for an $s p_{z}$ chain model.

remains almost the same. Notice that if we were to use two electrons per site, then the Fermi energy would lie in the gap, and the transmission would be zero. It must be noted, however, that the assumption of the WBL will break down if the Fermi energy is very close to a band edge.

Within this model of a gold chain, it is probably most physical to occupy the atoms with a single electron per site. However, in a more realistic description the $s$ and $p_{z}$ orbitals hybridize also with lower-energy $d$ orbitals. Also, the real systems are not translationally invariant, and charge neutrality need not be fully satisfied locally. Such things can complicate the picture enormously. Furthermore, the arbitrariness of the charge-neutrality procedure in the full spd model below renders the position of the Fermi energy with respect to the electronic structure of the wire somewhat uncertain. Thus, as we shall see, all of the cases shown in Fig. 5 are actually good
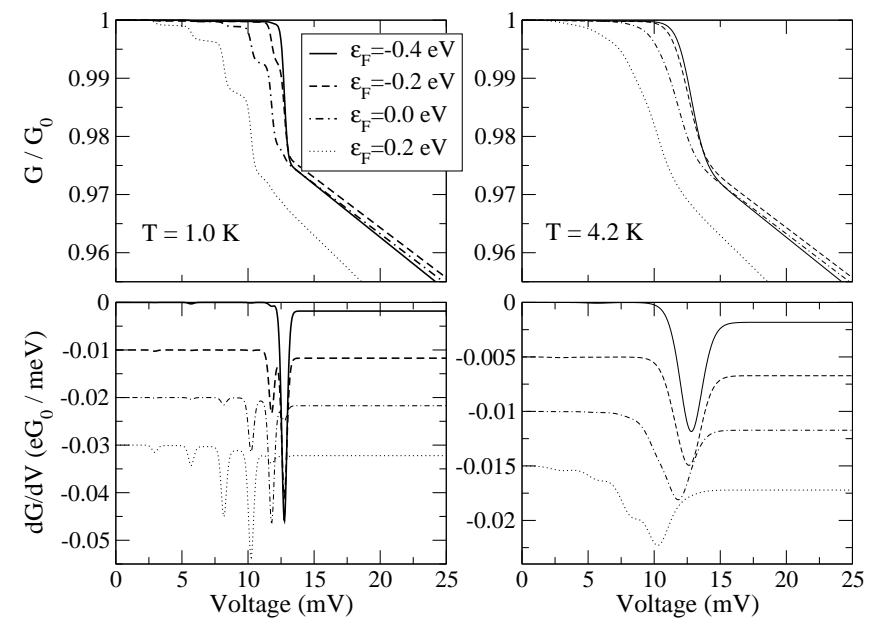

FIG. 5: The top panels show the conductance vs. voltage $G(V)$ for the $s p_{z}$ chain model with parameters as in Table and the lower panels show the derivative $\mathrm{d} G / \mathrm{d} V$. Here the curves have been shifted by integer multiples of 0.01 or 0.005 for clarity. The left panels are at $T=1.0 \mathrm{~K}$, while the right panels are at $T=4.2 \mathrm{~K}$. The vibrational modes are as in Fig. 1 of Ref. 20 .

descriptions of our results for the full spd model.

Although at low temperature there can be several peaks, at higher temperatures the steps fuse together, since the vibrational frequencies of the different modes are quite close to each other. The fusing effect of the steps would be further enhanced, if we introduced a larger broadening $\eta$ for the vibrational modes - here $\eta=0.002$ $\mathrm{meV} \ll k_{B} T$. The small $\eta$ also affects the results in another way: since the "lead coupling" of vibrations is small, the wire heats, and the distribution functions $N_{\alpha}$ differ strongly from the Bose distribution. The signature of this heating is the steep downward slope of the $G(V)$ curves at high voltage [19].

We have also tested the effect of $n$ 'th-nearest-neighbor hoppings and addition of nonorthogonality, but these have no essential qualitative effect on the results. These will be taken into account in the full spd parametrization, which we now turn to.

\section{FULL $s p d$ PARAMETRIZATION}

In this section we describe a more realistic tightbinding approach to the problem. We use the nineorbital spd parametrization of Papaconstantopoulos et al. 22, 23, 24, 25]. This type of spd TB approach is known to reproduce very well some nontrivial $a b$ initio results, like the numbers of conduction channels and the formation of zigzag Au chains [38, 39]. Thus we can be confident that the method gives at least good order-ofmagnitude estimates for all of the quantities which we shall be interested in.

However, since the parameters are extracted from firstprinciples bulk calculations, they cannot be exactly cor- 


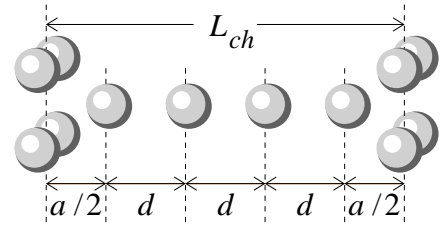

FIG. 6: Dimensions of the unoptimized geometry with $N_{c h}=$ 4. Here $a=4.08 \AA$, the fcc lattice constant. Only the coordinates of the $N_{c h}$ chain atoms are optimized.

rect for atomic point contacts, where the important atoms of the structure are significantly less coordinated than in bulk. It has thus become customary in the method to "correct" the parameters in the central cluster in order to satisfy local charge neutrality. Doing this typically brings the central cluster levels better in resonance with the lead orbitals. We compute the charge with the so-called Mulliken population analysis (see Appendix B), and only shift the on-site energies of the Hamiltonian. Tests with other ways of achieving neutrality give very similar results [28]. Furthermore, we have compared to results obtained without charge neutrality, and again find that there is not much qualitative difference, although charge neutrality yields conductances closer to $G_{0}$ on average. Also, without charge neutrality there appears to be a tendency for seeing lower-frequency vibrational modes in the conductance curves.

The results of this paper are generated using finite lead broadenings $\gamma_{L, R} \approx 1.0 \mathrm{eV}$. The limit $\gamma_{L, R} \rightarrow 0^{+}$can in principle be taken without affecting the results in any essential way.

\section{A. Geometry optimization and vibrational modes}

We consider two types of ideal geometries, the "A" and "B" ones, shown in Figs. 11 and 2] respectively. As mentioned, the leads are assumed to be of fcc type with the [001] axis in the transport (or $z$ ) direction. Before geometry optimization, the chain atoms are positioned as described in Fig. [6] The "length of the wire" $L_{c h}$ is defined as $L_{c h}=a+d\left(N_{c h}-1\right)$, where $N_{c h}$ is the number of atoms in the chain, $d$ the distance between them, and $a=4.08 \AA$ is the equilibrium lattice constant of the bulk fcc lattice. We only optimize the geometry of the $N_{c h}$ chain atoms - also in geometry B which has the "pyramids". Thus, although the interatomic distances change slightly from those of Fig. 6] $L_{c h}$ remains fixed.

To estimate the total energy $E\left(\vec{R}_{k}\right)$, we simply take a cluster which includes the wire and some atoms from the leads, solve for the electronic eigenstates $\epsilon_{\alpha}$, and then occupy the states according to charge neutrality. This energy, as a function of the $3 N_{c h}$ wire coordinates, is then optimized with standard library routines. As known previously [38], it is often energetically favorable for the gold chains to exist in a zigzag-like pattern instead of a linear one. Only after a sufficient amount of stretching (i.e., with a larger $d$ ) does the linear configuration become stable, after which it remains linear until the wire breaks. We find that the maximum $d$ at breaking is, depending on the geometry, typically something between $2.70-2.85$ $\AA$. There is no clear trend with increasing $N_{c h}$.

After the geometry is optimized, the energy function is used to compute the Hessian matrix $\mathcal{H}$. The eigenvalues $k_{\alpha}\left(\alpha=1, \ldots, 3 N_{c h}\right)$ are all positive, and the vibrational frequencies are simply given by $\omega_{\alpha}=\sqrt{k_{\alpha} / M}$. With both geometries, $\mathrm{A}$ and $\mathrm{B}$, we obtain quite similar vibrational frequencies and modes. For a linear wire, the modes can be classified as longitudinal or transverse in character. The highest-frequency modes are then always longitudinal ones, and the highest of them is of the ABL type [19].

\section{B. Elastic transmission}

Perhaps the most characteristic experimental property of gold chains is that they appear to have a conductance very close to the quantum of conductance $G_{0}$. Thus we shall briefly comment on the elastic transmission properties of the chains in our calculations. The present TB method was previously only used with bulk distances $d \approx 2.885 \AA$ between all the atoms [28]. In this case, we find very similar transmission functions $T_{0}(\epsilon)$ for our geometry B. These are often characterized by very long plateaus (of widths up to $2-3 \mathrm{eV}$ ) around the Fermi energy $\epsilon_{F}$, where $0.95 \lesssim T_{0} \lesssim 1.0$. The same is true for the results with and without charge neutrality.

However, when the geometry is optimized, the wide transmission plateaus close to one are replaced by larger oscillations. Still, at the Fermi energy, there is usually only a single open channel, which consists of $s, p_{z}$, and $d_{3 z^{2}-r^{2}}$ orbitals. Sometimes, a small contribution is seen arising from a second channel, involving the other $p$ and $d$ orbitals, as will be discussed below [28]. The transmission around $\epsilon_{F}$ varies between $0.7 \lesssim T_{0} \lesssim 1.0$. The present method is known to reproduce experimental conductance histograms rather well [40]. In particular, the conductance peak somewhat below $G / G_{0}=1$ is a very robust feature.

We determine $\epsilon_{F}$ by charge neutrality in the leads (or bulk). Tests with shifting its value from this position by $\sim 0.5 \mathrm{eV}$ (which simulates a gate effect) showed no significant qualitative effects on the results. The position of the Fermi energy with respect to the local electronic structure is still very important. This is because, besides the elastic transmission, the Fermi energy also fixes the "Fermi wavevector", which again determines what vibrational modes can be excited. In the present $\mathrm{TB}$ method, the use of bulk parameters and the charge neutrality procedure introduces some uncertainty in relation to this point. 


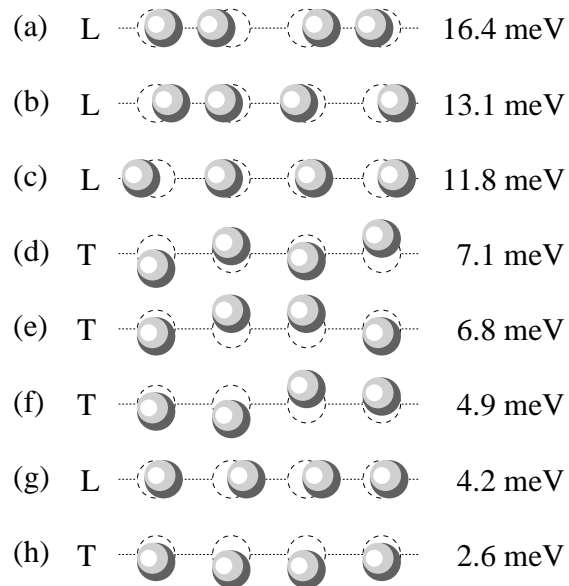

FIG. 7: A sketch of the vibrational eigenmodes of a linear four-atom gold wire, with energies corresponding to $d=2.62$ $\AA$ in geometry A. The longitudinal modes (L) are all nondegenerate, whereas the transverse modes $(\mathrm{T})$ are all doubly degenerate.

\section{Longitudinal and transverse modes}

Let us first discuss the basic observations using a simple example, namely, a linear chain of four atoms in geometry A. A schematic illustration of the vibrational modes is shown in Fig. 7 for $d=2.62 \AA$. There are four longitudinal modes and eight transverse modes. However, due to the fourfold rotation symmetry of the geometry around the axis of the wire, the transverse modes are all doubly degenerate. The zero-bias conductance is due to two partially open channels. The main contribution (about $98 \%$ of $\left.G_{0}\right)$ is due to a channel $\left(C_{1}\right)$ formed from $s$, $p_{z}$, and $d_{3 z^{2}-r^{2}}$ orbitals, which have the symmetry of the geometry. In addition, there is a small (less than $1 \%$ of $\left.G_{0}\right)$ contribution from a second, doubly degenerate channel $\left(C_{2}\right)$, which consists of $d_{x z}, d_{y z}, p_{x}$, and $p_{y}$ orbitals, which have a lower symmetry. Thanks to the symmetry of the $C_{1}$ channel, only longitudinal modes have a finite coupling constant in its subspace $\left(\boldsymbol{\lambda}_{C_{1}, C_{1}}^{\alpha}\right)$. In the subspace of the $C_{2}$ channel, also the transverse modes have a finite coupling $\left(\boldsymbol{\lambda}_{C_{2}, C_{2}}^{\alpha}\right)$. Thus we might expect that also the transverse modes give a small signal in the current.

Figure 8 shows an analysis of the contribution from the different modes to the differential conductance $G(V)=$ $\mathrm{d} I / \mathrm{d} V$. We divide this conductance into three parts according to the three current contributions: $G(V)=$ $G_{0} T_{0}+\delta G_{\text {inel }}(V)+\delta G_{e c}(V)$. Here $G_{0}=2 e^{2} / h, \delta G_{\text {inel }}=$ $\mathrm{d} I_{\text {inel }} / \mathrm{d} V$, and $\delta G_{e c}=\mathrm{d} \delta I_{e c} / \mathrm{d} V$. It is seen that $\delta G_{\text {inel }}$ gives always positive contributions to the conductance steps, while $\delta G_{e c}$ gives negative ones. As expected, we find that there is a finite step in both $\delta G_{\text {inel }}$ and $\delta G_{e c}$ due to all of the vibrational modes, also the transverse ones, although the latter are quite small. However, the contributions of $\delta G_{\text {inel }}$ and $\delta G_{e c}$ for the transverse modes almost perfectly cancel each other, such that only steps due to the longitudinal modes are seen in the total $G(V)$.

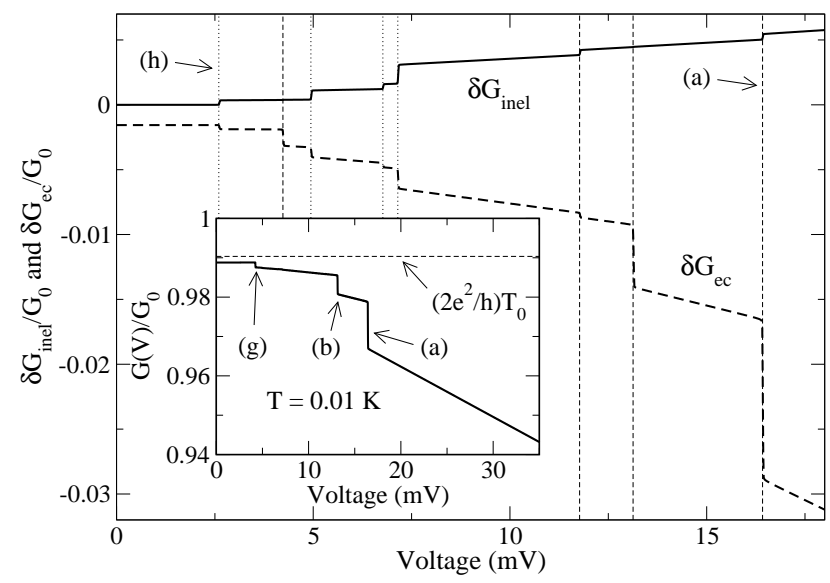

FIG. 8: Decomposition of the conductance $G(V)$ into $G_{0} T_{0}$, an "inelastic" contribution $\delta G_{\text {inel }}$, and an "elastic correction" $\delta G_{e c}$ for a four-atom wire. The geometry and the labels (a)(h) correspond to those of Fig. 7 Other parameters are $T=$ $0.01 \mathrm{~K}, \eta=0.002 \mathrm{meV}$. The solid step-like curve shows $\delta G_{\text {inel }}$, and the dashed one shows $\delta G_{e c}$. The increases of $\delta G_{i n e l}$ due to transverse modes are exactly canceled by decreases in $\delta G_{e c}$. The inset shows the elastic transmission (dashed line), and the total conductance $G(V)$ (solid line). In $G(V)$ only drops due to longitudinal modes are seen.

This cancellation is apparently due to the exact fourfold rotation symmetry, and the mirror symmetry with respect to the plane cutting the wire in the middle. In less symmetric geometries the transverse modes can also give finite contributions to $G(V)$.

In the case of zigzag wires, the distinction between longitudinal and transverse modes does not really exist, and all modes are always seen as steps in $G(V)$. An example of this is shown below. Furthermore, if the elastic transmission $T_{0}$ is very small, then also the transport coefficients $T_{\alpha}^{e c}$ and $T_{\alpha}^{e c L R}$ tend to be small, since they all depend on the matrix $\tilde{\boldsymbol{G}}^{r} \boldsymbol{\Gamma}_{R} \tilde{\boldsymbol{G}}^{a} \boldsymbol{\Gamma}_{L}$. In this way, for example, it may also be possible to have large positive steps in $G(V)$, but we never see them for the charge-neutral gold wires. For other materials, the situation may be different.

Thus, we find that the conductance features depend in an intricate way on the symmetries of the geometry, the symmetries of the vibrational modes, the coupling constants, as well as the symmetries of the electronic states which are relevant at the Fermi energy.

\section{Conductance curves of linear wires}

Here we discuss in more detail, how our conductancevoltage curves for linear wires look like. Figure 9 shows an example for geometry A with a wire of $N_{c h}=4$ atoms while Fig. 10 is for a wire of $N_{c h}=11$ atoms in geometry B. In both figures, the left-hand panels are calculated at $T=4.2 \mathrm{~K}$ and with a small $\eta$, such that they are more or less in the externally undamped regime. The 

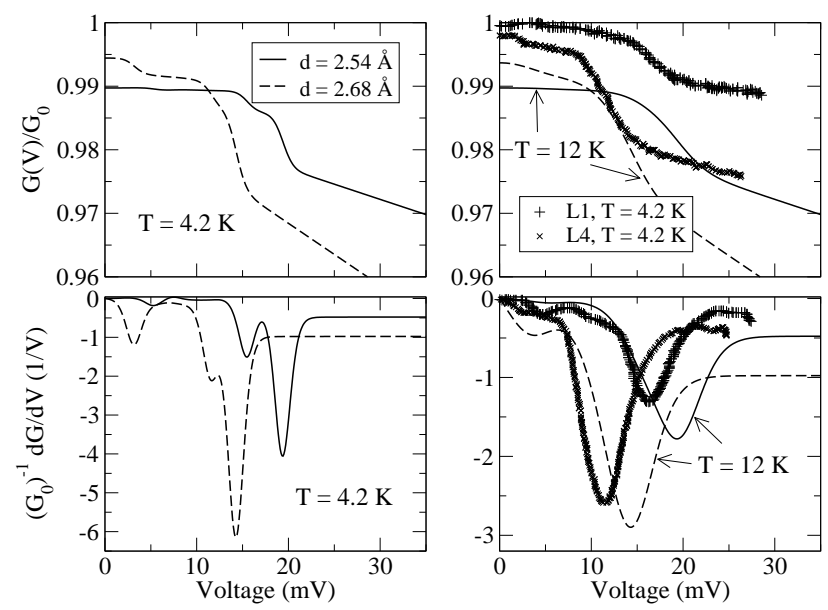

FIG. 9: Comparison between theory and experiment for $N_{c h}=4$ in geometry $\mathrm{A}$. The solid and dashed curves correspond to theoretical results for $d=2.54 \AA$ and $d=2.68 \AA$, respectively. On the left-hand panels $T=4.2 \mathrm{~K}$, whereas on the right-hand panels these curves have been broadened with a larger temperature $T=12 \mathrm{~K}$; in both cases $\eta=0.02 \mathrm{meV}$. The experimental results $\mathrm{L} 1(+)$ and $\mathrm{L} 4(\times)$ correspond to the notation and results of Fig. $1(\mathrm{~d})$ of Ref. 18 with $V>0$. They are obtained for a 7 -atom chain at $T=4.2 \mathrm{~K}$.
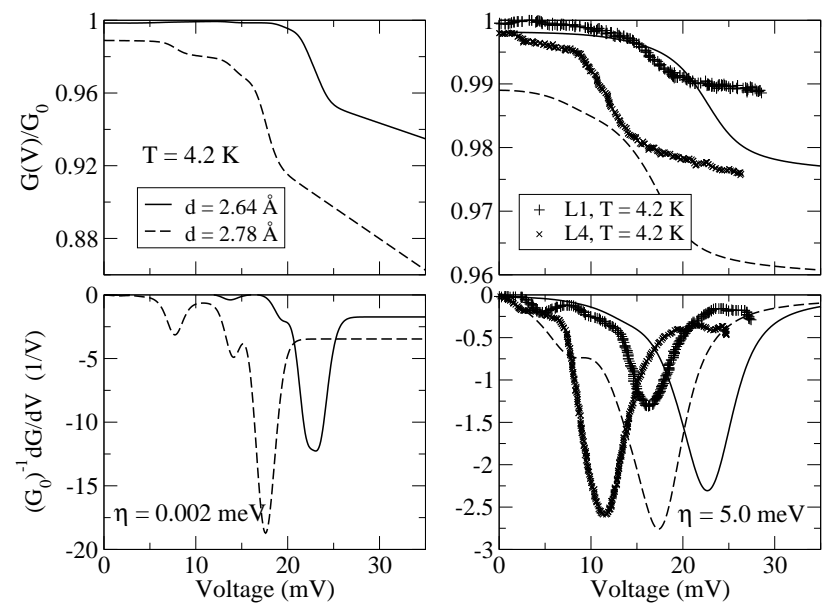

FIG. 10: Comparison between theory and experiment for $N_{c h}=11$ in geometry B. All results are at $T=4.2 \mathrm{~K}$. The solid and dashed curves correspond to theoretical results for $d=2.64 \AA$ and $d=2.78 \AA$, respectively. On the left-hand panels $\eta=0.002 \mathrm{meV}$, whereas on the right-hand panels the curves have been broadened with a bath-coupling $\eta=5.0$ $\mathrm{meV}$. The experimental results $\mathrm{L} 1(+)$ and $\mathrm{L} 4(\times)$ are as in Fig. 9

right-hand panels show two examples of the experimental results for a wire of approximately 7 atoms taken at the temperature $T=4.2 \mathrm{~K}[18]$. Comparing these to the theoretical curves on the left-hand side, one immediately notices that if the conductance drop is to be due to a single mode, then the $\sim 5 \mathrm{meV}$ width of the peak in the experimental $\mathrm{d} G / \mathrm{d} V$ cannot be explained by temperature alone [19]. On the other hand, the energy distance

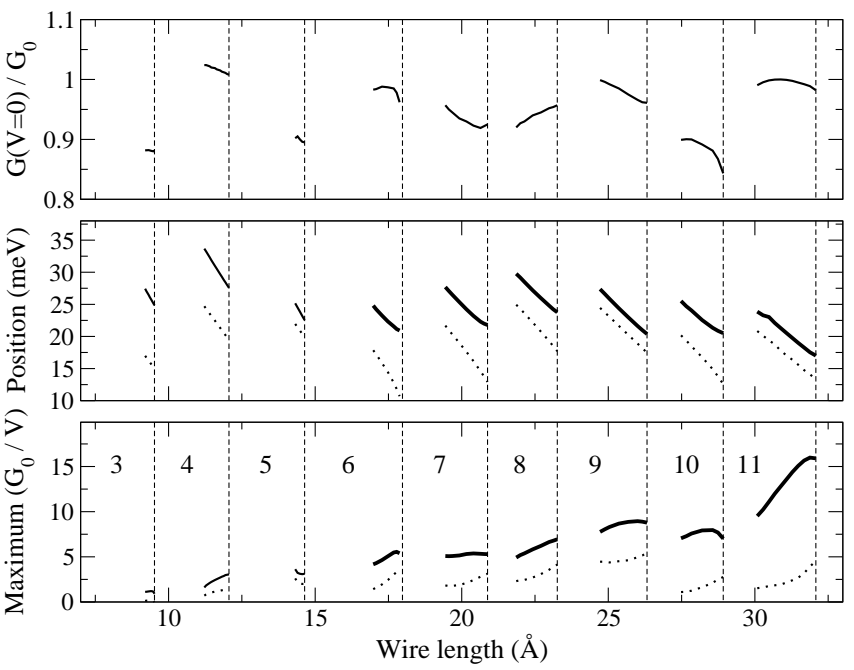

FIG. 11: Zero-bias conductances, voltage positions $V_{p h}$, and the local maxima of $|\mathrm{d} G / \mathrm{d} V|$ for different wire lengths $L_{c h}$ and atom numbers $N_{c h}$, indicated by the numbers. The results are obtained for linear wires in geometry $\mathrm{B}$, with $\eta=0.02 \mathrm{meV}$. The dashed lines show the limits beyond which the wire breaks upon further stretching. On the left side of each curve, the wire has a non-linear form, and the peak structure may be different (see text). The solid lines correspond to the main peaks and the dotted lines to the second-largest ones. Thickening of the lines indicates that the peak consists of two close-lying modes.

between the vibrational modes is rather large $\gtrsim k_{B} T$, so that at $T=4.2 \mathrm{~K}$, a peak consisting of several subpeaks can usually be easily recognized. For example, the highest-frequency peak in Fig. [10]actually consists of two peaks, and it is still not wide enough. Also in Fig.9 there are at least three separate peaks visible.

Thus we conclude that in the experiment there are probably other broadening mechanisms at play besides temperature. In the right panels of Fig. 9 we compare the experiment with a theoretical result broadened by a higher temperature, while in the right panels of Fig. 10 we use the parameter $\eta$ to broaden the peaks. In the latter, the system is already in the externally damped regime, with very little local heating: in addition to the broadening, the damping is signified by a smaller slope after the drop. In either case, the peaks due to individual modes are smoothed out to form a single one, with a width comparable to that seen in experiments. In this way, it is possible to obtain a rather good quantitative correspondence between theory and experiment.

We have also studied systematically how the main features of the conductance-voltage curves vary when linear chains with atom numbers $3 \leq N_{c h} \leq 11$ are stretched. The results for $T=4.2 \mathrm{~K}$ and a small $\eta$ are plotted in Fig. 11. Here we show the zero-bias conductance $G(V=0)$, the voltage positions $V_{p h}$ of the main peaks in $\mathrm{d} G / \mathrm{d} V$, and the corresponding local maximum values of $|\mathrm{d} G / \mathrm{d} V|$. The peak height is not the same as the peak area which is plotted in Ref. 18, but is roughly proportional to it, since 
the width of the peaks is $\sim k_{B} T$, where the temperature is $T=4.2 \mathrm{~K}$.

In this figure, many features can be recognized. The $G(V=0)$ values fluctuate between 0.85 and 1.0 , but there is no clear "parity effect" — or at least the effect appears to reverse its direction after $N_{c h}=8$. The present method most likely does not describe such parity effects correctly. However, also different ab initio approaches are known to give conflicting results [7]. In some calculations, the transmission has been found to oscillate also with the stretching of a wire with fixed $N_{c h}[12$.

The positions of the peaks move to lower voltages when the wire is stretched, as expected from the "softening" of the bonds, and the resulting decrease in the vibrational frequencies. There is also a clear trend toward lower frequencies with increasing $N_{c h}$. These findings are similar to what is seen in the experiment [18]. Also similarly, the peak heights increase with stretching, and with increasing $N_{c h}$, although the increase with wire length $L_{c h}$ is not obviously linear as for the simple chain models of Sec. $\mathbb{\nabla}[20$. There is also a correlation between the zero-bias conductances and the peak heights: when the conductance is low, also the stretching behavior is rather anomalous (in particular in the cases of 5 and 10 atoms.)

The most visible difference between the results of Fig. 111 and the experiment is that we consistently see signatures of several vibrational modes: typically there are two peaks visible in $\mathrm{d} G / \mathrm{d} V$. However, the higher peak is always at a larger voltage and, as the number of atoms $N_{c h}$ grows, the secondary peaks become less and less significant. For example, for 11 atoms there is essentially only a single peak visible (see Fig. 101). This, however, is due to two close-lying modes: the highest-frequency "ABL" mode, and the one next to it in frequency. As explained above, this discrepancy of several peaks can be corrected by increasing the parameter $\eta$. Note also that this behavior was already present in the chain model of Sec. [V]

We also see that the largest conductance drops are systematically at too high voltages compared to experimental values $V_{p h} \approx 10-20 \mathrm{mV}$ for a 7 -atom chain. This is not surprising, given the simplified way in which we compute the vibrational modes. The frequencies of the vibrational modes might be lowered, if we also allowed for the motion of atoms outside of the chain. In other words, it is possible that the lead coupling, done in a proper way, would lead to a "redshift" of the frequencies. As noted above, the electron-vibration coupling gives such a redshift 29], but this effect may be too small to explain the discrepancy.

Although the steps in conductance are almost always downward when $G(V=0)$ is close to $G_{0}$, sometimes also weak increases in the conductance at low voltage can be seen. These appear to be related to the longitudinal "center-of-mass" mode. For linear chains, we do not find any significant contribution from transverse modes, as explained previously.
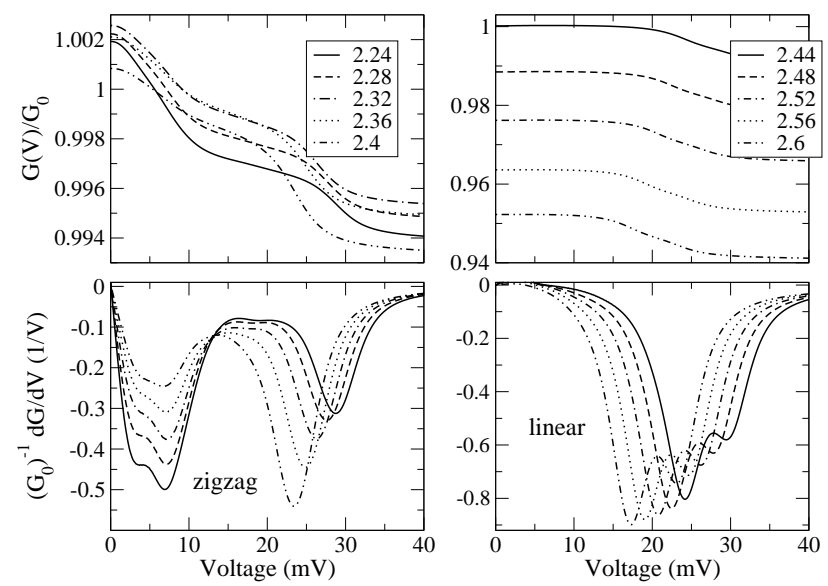

FIG. 12: The difference between zigzag and linear wires of 6 atoms in geometry $\mathrm{A}$ at $T=4.2 \mathrm{~K}$ and with $\eta=5.0 \mathrm{meV}$. The numbers in the legend indicate the parameter $d / \AA$, which describes the amount of stretching. In the left panels the wire has a zigzag character, while in the right panels it is linear. In case of the zigzag-wire, practically all of the vibrational modes contribute to the observed steps, but they form a clear doublestep structure. In the linear wire, the elastic transmission varies a lot under stretching in this example.

\section{E. Zigzag wires}

We have also studied briefly the zigzag chains shown in Fig. 11 Figure 12 shows a comparison between the signatures of a zigzag chain and a linear chain in the conductance-voltage curves $G(V)$. In this example, a chain of $N_{c h}=6$ atoms in geometry A, and a large broadening of the vibrational modes $(\eta=5.0 \mathrm{meV})$ was used. However, very similar results were found for various different atom numbers. For small values of $d$, the wire has a zigzag character, but at $d \approx 2.42 \AA$, the wire becomes linear.

For the zigzag chain, there are two well-separated (series of) conductance drops. The one at low bias is higher for small $d$ 's. As the chain is stretched, the height of the lower-frequency step decreases, while that of the higherfrequency one increases. For the linear chain, there is essentially only one high-voltage drop, which is actually due to two different longitudinal modes. In the case of a zigzag wire, the vibrational modes have complicated symmetries, and practically all of them are contributing to the current steps. However, here their identities are completely smeared out due to the broadening. This calculation provides a clear prediction of how the zigzagto-linear transition may be seen in experiments.

\section{CONCLUSIONS AND DISCUSSION}

We have studied the onset of dissipation by excitation of vibrational modes in atomic gold wires, using a tightbinding model. We have studied in a systematic way 
how the stretching of wires with different atomic numbers affects the conductance steps, and find a reasonable agreement with experiments. Previously such a study has only been done within simple chain models [20]. We have also considered two different geometries, which yield qualitatively similar results. Finally, we studied the conductance signatures of zigzag-type wires, and predict a double-step structure in contrast to the single step of linear chains.

Our results for the linear chains agree rather well with experiments and previous ab initio calculations, apart from the incomplete "mode selectivity". In this context, we have pointed out the importance of taking into account the broadening of vibrational modes due to their coupling to the leads, especially in the limit where the vibrational mode distribution is assumed to be strongly damped. We derived equations in the wide-band limit, which take this into account in a phenomenological manner. The wide-band limit combined with the lowest-order perturbation approach (presented in Sec. IV] and Appendix E) appears to provide a sufficiently good description of the phenomenology of electron-vibration interaction in atomic wires. To make further progress, more detailed calculations of the lead-coupling of the vibrational modes are needed.

As explained above, the condition which determines what modes yield conductance drops is essentially that of momentum conservation. We find numerically that the momentum-conservation idea works well also for finite wires with 10 atoms or more. However, in shorter wires, the electronic structure is very complicated, and the connection between symmetries of excited modes and those of the electronic states is hard to analyze.

Even if there is approximate momentum conservation, there is no fundamental reason why only a single mode would be excited, or that the highest-frequency (or other ABL) modes should necessarily be involved. This remains true also for very long wires, since at the same time when the momentum conservation becomes more and more accurately satisfied, also the energy density (and hence momentum-space density) of the modes increases. It is thus more likely that a large "wave packet" of several nearby phonon modes is always excited. However, the charge neutrality of the wire indeed appears to be favoring the highest-frequency modes, just as predicted by the simple chain models discussed in Sec. $\nabla$ Nevertheless, it is important to note that small differences in details of the geometry or the implementation may affect numerical values of results significantly. The band structure of an infinite, linear gold wire is already quite complicated [28, 38], such that a small error in the Fermi energy, and hence of the Fermi wavevector, will immediately shift the resonance to slightly different vibrational modes.

Studying different materials (Pt and Ir) with this same method would be straightforward in principle. However, it seems that the parameters available for these materials are not very good for geometry optimizations of the wires. This is because the overlap matrices easily lose their positive definiteness when the validity range of the parametrization is exceeded (with the gold parameters 25], such problems never appeared). Thus, as already implied in Ref. 28, one should probably use more general $a b$ initio methods, or at least parameters which have been specifically fit to work for chain geometries with a large span of interatomic distances. Compared to $a b i n i-$ tio methods, TB still has the clear advantage of being computationally efficient.

\section{Acknowledgments}

It is a pleasure to acknowledge useful discussions with N. Agrait, M. Brandbyge, T. Frederiksen, A. LevyYeyati, A. Martin-Rodero and G. Rubio. This work was financially supported by the Helmholtz Gemeinschaft within the Nachwuchsgruppe program (contract VH-NG029) and by the DFG within the Center for Functional Nanostructures.

\section{APPENDIX A: CALCULATION OF THE COUPLING MATRIX ELEMENTS}

Here we describe our method of computing the matrix elements $M_{i j}^{k \mu}=\left\langle i\left|\nabla_{k \mu} H\right| j\right\rangle$, where the derivative is with respect to the components $\mu=x, y, z$ of the ionic coordinates $\vec{R}_{k}$. In an implementation making use of TB parametrization, one has no direct access to either the basis states or $|i\rangle$, or the Hamiltonian $H$ - only the representation $H_{i j}=\langle i|H| j\rangle$ and the overlap matrix $S_{i j}=\langle i \mid j\rangle$ as a function of $\vec{R}_{k}$ are known. Thus, the matrix elements must be calculated more indirectly. Below we sketch a derivation, which follows, in some sense, the ideas of Ref. 41. The derivation is not exact, as we only consider an isolated central cluster.

Let $|i\rangle,|j\rangle, \ldots$ denote the atomic orbital (AO) basis states, and $|\alpha\rangle,|\beta\rangle, \ldots$ the electronic eigenstates (molecular orbitals, MO) of the central-cluster electronic Hamiltonian $H$. The eigenstates satisfy

$$
\begin{aligned}
& H|\alpha\rangle=\epsilon_{\alpha}|\alpha\rangle, \\
& \langle\alpha \mid \beta\rangle=\delta_{\alpha \beta} .
\end{aligned}
$$

If the expansion of the MO's in the AO basis is denoted $|\alpha\rangle=\sum_{i}|i\rangle C_{i \alpha}$, where $C_{i \alpha}=\left(\mathbf{c}_{\alpha}\right)_{i}$ then we have the matrix equations

$$
\begin{aligned}
\boldsymbol{H} \mathbf{c}_{\alpha} & =\boldsymbol{S} \mathbf{c}_{\alpha} \epsilon_{\alpha} \\
\mathbf{c}_{\alpha}^{\dagger} \boldsymbol{S} \mathbf{c}_{\beta} & =\delta_{\alpha \beta} .
\end{aligned}
$$

Let us also note the form of the completeness relation of the MO's $\sum_{\alpha}|\alpha\rangle\langle\alpha|=1$ in the AO basis: $\sum_{\alpha} \mathbf{c}_{\alpha} \mathbf{c}_{\alpha}^{\dagger}=$ $\boldsymbol{S}^{-1}$, while for the AO's themselves $\sum_{i j}|i\rangle\left(\boldsymbol{S}^{-1}\right)_{i j}\langle j|=$ 1. Since the basis states also move with the ions, we should write more carefully $|i(Q)\rangle,|\alpha(Q)\rangle, H(Q), H_{i j}(Q)$ 
etc., where $Q$ is a shorthand for the ionic displacements $\vec{Q}_{k}=\vec{R}_{k}-\vec{R}_{k}^{(0)}$. The point in the electron-vibration coupling is that moving an ion will induce a perturbation of the form

$$
\begin{aligned}
\boldsymbol{H} & \rightarrow \boldsymbol{H}+\boldsymbol{H}^{\prime} Q \\
\boldsymbol{S} & \rightarrow \boldsymbol{S}+\boldsymbol{S}^{\prime} Q
\end{aligned}
$$

and one can calculate its effect on the state vectors $\mathbf{c}_{\alpha}$ and eigenenergies $\epsilon_{\alpha}$ by means of simple first-order perturbation theory. Here " I" denotes a derivative with respect to $Q$.

Let us expand the basis states as $|i(Q)\rangle \approx|i\rangle+\left|i^{\prime}\right\rangle Q$, where $|i\rangle$ now denote the unperturbed basis. The matrix element which we are looking for is really the quantity

$$
M_{i j}=\left.\left\langle i\left|H^{\prime}(Q)\right| j\right\rangle\right|_{Q=0}
$$

This may be obtained by considering the expansion of $\langle i|H(Q)| j\rangle$ in $Q$, since

$$
\langle i|H(Q)| j\rangle=H_{i j}(0)+\left.\left\langle i\left|H^{\prime}(Q)\right| j\right\rangle\right|_{Q=0} Q .
$$

By inserting $1=\sum_{\alpha}|\alpha(Q)\rangle\langle\alpha(Q)|$ one easily finds

$$
\langle i|H(Q)| j\rangle=\sum_{\alpha}\langle i \mid \alpha(Q)\rangle \epsilon_{\alpha}(Q)\langle\alpha(Q) \mid j\rangle .
$$

Then, by inserting $|\alpha(Q)\rangle=\sum_{i}|i(Q)\rangle C_{i \alpha}(Q)$, expanding $\epsilon_{\alpha}(Q) \approx \epsilon_{\alpha}+\epsilon_{\alpha}^{\prime} Q, C_{i \alpha}(Q) \approx C_{i \alpha}+C_{i \alpha}^{\prime} Q$, and equating terms linear in $Q$

$$
\begin{aligned}
M_{i j} & =\sum_{\alpha} \sum_{k l}\left\{S_{i k} C_{k \alpha} \epsilon_{\alpha}^{\prime} C_{l \alpha}^{*} S_{l j}\right. \\
& +S_{i k}^{\prime(2)} C_{k \alpha} \epsilon_{\alpha} C_{l \alpha}^{*} S_{l j}+S_{i k} C_{k \alpha} \epsilon_{\alpha} C_{l \alpha}^{*} S_{l j}^{\prime(1)} \\
& \left.+S_{i k} C_{k \alpha}^{\prime} \epsilon_{\alpha} C_{l \alpha}^{*} S_{l j}+S_{i k} C_{k \alpha} \epsilon_{\alpha} C_{l \alpha}^{\prime *} S_{l j}\right\}
\end{aligned}
$$

Here we introduced the one-sided overlap derivatives $\left[\boldsymbol{S}^{\prime(1)}\right]_{i j}=\left\langle i^{\prime} \mid j\right\rangle,\left[\boldsymbol{S}^{\prime(2)}\right]_{i j}=\left\langle i \mid j^{\prime}\right\rangle$, which satisfy $\boldsymbol{S}^{\prime}=$ $\boldsymbol{S}^{\prime(1)}+\boldsymbol{S}^{\prime(2)}$, and $\boldsymbol{S}^{\prime(2)}=\left[\boldsymbol{S}^{\prime(1)}\right]^{\dagger}[41]$.

The result of Eq. (A7) may be simplified considerably by inserting the expressions for $\epsilon_{\alpha}^{\prime}$ and $C_{i \alpha}^{\prime}$, which are easily derived. First, if we denote

$$
\begin{aligned}
M_{\alpha \beta} & \equiv \sum_{k l} C_{k \alpha}^{*} M_{i j} C_{l \beta}, \quad H_{\alpha \beta}^{\prime} \equiv \sum_{k l} C_{k \alpha}^{*} H_{i j}^{\prime} C_{l \beta}, \\
S_{\alpha \beta}^{\prime(1,2)} & \equiv \sum_{k l} C_{k \alpha}^{*} S_{i j}^{\prime(1,2)} C_{l \beta}
\end{aligned}
$$

and so on, one may show that

$$
M_{\alpha \beta}=H_{\alpha \beta}^{\prime}-S_{\alpha \beta}^{\prime(1)} \epsilon_{\beta}-\epsilon_{\alpha} S_{\alpha \beta}^{\prime(2)}
$$

Then, using the completeness relations $\sum_{\alpha} \sum_{k} S_{i k} C_{k \alpha} C_{j \alpha}^{*}=\sum_{\alpha} \sum_{k} C_{i \alpha} C_{k \alpha}^{*} S_{k j}=\delta_{i j}$ one may transform back to the AO basis:

$$
\begin{aligned}
& M_{i j}=H_{i j}^{\prime}-\sum_{\alpha} \sum_{k l}\{ \\
& \left.S_{i k}^{\prime(1)} C_{k \alpha} \epsilon_{\alpha} C_{l \alpha}^{*} S_{l j}+S_{i k} C_{k \alpha} \epsilon_{\alpha} C_{l \alpha}^{*} S_{l j}^{\prime(2)}\right\} .
\end{aligned}
$$

This is the final expression for the matrix elements. The overlap corrections due to the nonorthogonal basis often turn out to be rather small in practice, an the orthogonal result $\boldsymbol{M}=\boldsymbol{H}^{\prime}$ is a reasonable first approximation. The presence of the corrections tends to make the conductance steps slightly larger.

\section{APPENDIX B: POPULATION ANALYSIS AND DERIVATION OF THE CURRENT FORMULA}

Although the expression for the current is well known, its derivation in the nonorthogonal basis is not entirely trivial [42]. The current is the time derivative of charge transported from $L$ to $C$ and on to $R$. However, while the total charge of the full system is well defined, the partial charges of the distinct regions are not - instead, there are different ways of doing "population analysis". To be more self-contained, we present here a compact discussion of these issues.

Let us consider, for simplicity, a single orbital per atomic site $i$. The total charge (particle number, actually) is given by

$$
Q=\langle\hat{Q}\rangle=\sum_{j, k} P_{j k} S_{k j}
$$

where $\hat{Q}=\sum_{j, k} d_{k}^{\dagger} S_{k j} d_{j}$ and we define the density ma$\operatorname{trix} P_{j k}=\left\langle d_{k}^{\dagger} d_{j}\right\rangle$. Partial charges may be introduced for example with the following "Mulliken" population analysis 43

$$
\begin{aligned}
Q & =Q_{L}+Q_{C}+Q_{R}=\sum_{j \in L, k} P_{j k} S_{k j} \\
& +\sum_{j \in C, k} P_{j k} S_{k j}+\sum_{j \in R, k} P_{j k} S_{k j} .
\end{aligned}
$$

One may also define the quantities

$$
Q_{\Omega}^{\prime}=\left\langle\hat{Q}_{\Omega}^{\prime}\right\rangle=\sum_{j, k \in \Omega} P_{j k} S_{k j},
$$

where $\hat{Q}_{\Omega}^{\prime}=\sum_{j, k \in \Omega} d_{k}^{\dagger} S_{k j} d_{j}$, and $\Omega=L, C, R, C+$ $R, C+L$. Here $C+L(C+R)$ refers to the combined system involving $C$ and $L$ ( $C$ and $R$ ) regions. The charges $Q_{L, C, R}^{\prime}$ are good approximations to $Q_{L, C, R}$ if the regions are all large, since the corrections are proportional to the surface area of the interfaces.

What we want is the particle current $\hat{J}$ through a boundary between two regions of space, $L$ and $C$, for example. We expect it to satisfy

$$
2 \hat{J}=\frac{\partial}{\partial t}\left(\hat{Q}_{L}^{\prime}-\hat{Q}_{C+R}^{\prime}\right)
$$

Let us rewrite $\hat{Q}_{\Omega}^{\prime}=\hat{N}_{\Omega}+\sum_{j, k \in \Omega, j \neq k} d_{k}^{\dagger} S_{k j} d_{j}$, where $\hat{N}_{\Omega}=\sum_{j \in \Omega} \hat{N}_{j}$ and $\hat{N}_{j}=d_{j}^{\dagger} d_{j}$ for $\Omega=L, C+R$. 
Now, in the Heisenberg picture the $d_{j}$ operators satisfy the equations of motion $\mathrm{i} \hbar \dot{d}_{j}=\left[d_{j}, \hat{H}_{e}\right]$ and hence $\mathrm{i} \hbar \frac{\partial}{\partial t} \sum_{k} S_{j k} d_{k}=\sum_{k} H_{j k} d_{k}$. Using ideas similar to Ref. 44, we find

$$
\frac{\partial}{\partial t} \hat{N}_{j}=d_{j}^{\dagger} \sum_{k \neq j} \frac{1}{\mathrm{i} \hbar}\left(H_{j k}-S_{j k} \mathrm{i} \hbar \frac{\partial}{\partial t}\right) d_{k}+\text { h.c. }
$$

Next, considering the following quantity, we notice that all hopping contributions except $\boldsymbol{H}_{L C}$ and $\boldsymbol{H}_{L R}$ cancel:

$$
\begin{aligned}
\frac{\partial}{\partial t} & \left(\hat{N}_{L}-\hat{N}_{C+R}\right) \\
& =\sum_{j \in L, k \in C+R} d_{j}^{\dagger} \frac{1}{\mathrm{i} \hbar}\left(H_{j k}-S_{j k} \mathrm{i} \hbar \frac{\partial}{\partial t}\right) d_{k}+\text { h.c. } \\
& -\sum_{j \in C+R, k \in L} d_{j}^{\dagger} \frac{1}{\mathrm{i} \hbar}\left(H_{j k}-S_{j k} \mathrm{i} \hbar \frac{\partial}{\partial t}\right) d_{k}+\text { h.c. } \\
& -\sum_{j, k \in L, k \neq j} d_{j}^{\dagger} S_{j k} \frac{\partial}{\partial t} d_{k}+\text { h.c. } \\
& +\sum_{j, k \in C+R, k \neq j} d_{j}^{\dagger} S_{j k} \frac{\partial}{\partial t} d_{k}+\text { h.c. }
\end{aligned}
$$

When the last two overlap terms are moved to the lefthand side, it becomes exactly what we called $2 \hat{J}$. Thus the expectation value is

$$
\begin{aligned}
2 J & =2\langle\hat{J}\rangle \\
& =\left.\sum_{j \in L, k \in C+R} \frac{1}{\mathrm{i} \hbar}\left(H_{j k}-S_{j k} \mathrm{i} \hbar \frac{\partial}{\partial t}\right)\left\langle d_{j}^{\dagger}\left(t^{\prime}\right) d_{k}(t)\right\rangle\right|_{t^{\prime}=t} \\
& -\left.\sum_{j \in C+R, k \in L} \frac{1}{\mathrm{i} \hbar}\left(H_{j k}-S_{j k} \mathrm{i} \hbar \frac{\partial}{\partial t}\right)\left\langle d_{j}^{\dagger}\left(t^{\prime}\right) d_{k}(t)\right\rangle\right|_{t^{\prime}=t} \\
& + \text { c.c. }
\end{aligned}
$$

Now, assuming that $\boldsymbol{H}_{L R}=\boldsymbol{S}_{L R}=0$ and using $\mathrm{i}\left\langle d_{j}^{\dagger}\left(t^{\prime}\right) d_{k}(t)\right\rangle=G_{k j}^{+-}\left(t, t^{\prime}\right)$, we finally have

$$
\begin{aligned}
J(t) & =-\frac{1}{\hbar} \operatorname{Re} \operatorname{Tr}\left[\left(\boldsymbol{H}_{L C}-\boldsymbol{S}_{L C} \mathrm{i} \hbar \frac{\partial}{\partial t}\right) \boldsymbol{G}_{C L}^{+-}\left(t, t^{\prime}\right)\right. \\
& \left.-\left(\boldsymbol{H}_{C L}-\boldsymbol{S}_{C L} \mathrm{i} \hbar \frac{\partial}{\partial t}\right) \boldsymbol{G}_{L C}^{+-}\left(t, t^{\prime}\right)\right]\left.\right|_{t^{\prime}=t} .
\end{aligned}
$$

The charge current is then obtained as $I=-2 e J$, where the factor 2 is the spin degeneracy. An analogous derivation may be carried out for the current over the $C$ - $R$ boundary.

In stationary state all the propagators only depend on the time difference $t-t^{\prime}$, and this result may be Fourier transformed into an energy representation, where one has to replace $\mathrm{i} \hbar \partial / \partial t \rightarrow \epsilon$.

\section{APPENDIX C: NEGF FORMALISM: TECHNICAL DETAILS}

Our notation differs slightly from what is the standard. In particular, our functions $G^{ \pm \mp}$ are equal to the $G^{\lessgtr}$ functions of Refs. 19, 32, for example. Let us write the definitions for the most important electron propagators

$$
\begin{aligned}
G_{i j}^{r}\left(t, t^{\prime}\right) & =-\mathrm{i}\left\langle\left\{d_{i}(t), d_{j}^{\dagger}\left(t^{\prime}\right)\right\}\right\rangle \theta\left(t-t^{\prime}\right) \\
G_{i j}^{a}\left(t, t^{\prime}\right) & =\mathrm{i}\left\langle\left\{d_{i}(t), d_{j}^{\dagger}\left(t^{\prime}\right)\right\}\right\rangle \theta\left(t^{\prime}-t\right) \\
G_{i j}^{+-}\left(t, t^{\prime}\right) & =\mathrm{i}\left\langle d_{j}^{\dagger}\left(t^{\prime}\right) d_{i}(t)\right\rangle \\
G_{i j}^{-+}\left(t, t^{\prime}\right) & =-\mathrm{i}\left\langle d_{i}(t) d_{j}^{\dagger}\left(t^{\prime}\right)\right\rangle .
\end{aligned}
$$

Similar expressions hold for phonons, but with the replacement $\{\cdot, \cdot\} \rightarrow[\cdot, \cdot]$. Below, all Green functions appear Fourier transformed with respect to $t-t^{\prime}$ into an energy representation.

\section{Electron propagators and self-energies}

All expressions for the relevant electron Green functions follow from the "Dyson" and "Keldysh" equations

$$
\begin{aligned}
\boldsymbol{G}^{r} & =\left[\left(\boldsymbol{g}^{r}\right)^{-1}-\boldsymbol{\Sigma}^{r}\right]^{-1} \\
\boldsymbol{G}^{ \pm \mp} & =\left(1+\boldsymbol{G}^{r} \boldsymbol{\Sigma}^{r}\right) \boldsymbol{g}^{ \pm \mp}\left(1+\boldsymbol{\Sigma}^{a} \boldsymbol{G}^{a}\right)-\boldsymbol{G}^{r} \boldsymbol{\Sigma}^{ \pm \mp} \boldsymbol{G}^{a},
\end{aligned}
$$

where the upper or lower signs can be chosen. Here the $\boldsymbol{g}$ denote the Green functions for an uncoupled central part in the absence of electron-vibration interactions and $\boldsymbol{\Sigma}^{r, \pm \mp}$ are the sums of all self-energies containing the effects of both (see below). The uncoupled functions are

$$
\begin{aligned}
\boldsymbol{g}^{r} & =\left[\epsilon \boldsymbol{S}_{C C}+\mathrm{i} \gamma_{c} / 2-\boldsymbol{H}_{C C}\right]^{-1} \\
\boldsymbol{g}^{+-} & =-f_{C}\left(\boldsymbol{g}^{r}-\boldsymbol{g}^{a}\right) \\
\boldsymbol{g}^{-+} & =-\left(f_{C}-1\right)\left(\boldsymbol{g}^{r}-\boldsymbol{g}^{a}\right) .
\end{aligned}
$$

Here $f_{C}$ is the equilibrium Fermi distribution. Note that $\gamma_{C}=\mathrm{i}\left[\left(\boldsymbol{g}^{a}\right)^{-1}-\left(\boldsymbol{g}^{r}\right)^{-1}\right]$, which is an infinitesimal quantity.

The functions where the lead coupling is taken into account but electron-vibration coupling is still neglected are given by

$$
\begin{aligned}
\tilde{\boldsymbol{G}}^{r} & =\left[\epsilon \boldsymbol{S}_{C C}-\boldsymbol{H}_{C C}-\boldsymbol{\Sigma}_{L}^{r}-\boldsymbol{\Sigma}_{R}^{r}\right]^{-1} \\
\tilde{\boldsymbol{G}}^{+-} & =-\boldsymbol{G}^{r}\left(\boldsymbol{\Sigma}_{L}^{+-}+\boldsymbol{\Sigma}_{R}^{+-}-\mathrm{i} \boldsymbol{\gamma}_{C} f_{C}\right) \boldsymbol{G}^{a} \\
\tilde{\boldsymbol{G}}^{-+} & =-\boldsymbol{G}^{r}\left[\boldsymbol{\Sigma}_{L}^{-+}+\boldsymbol{\Sigma}_{R}^{-+}-\mathrm{i} \boldsymbol{\gamma}_{C}\left(f_{C}-1\right)\right] \boldsymbol{G}^{a} .
\end{aligned}
$$

and $\tilde{\boldsymbol{G}}^{a}=\left(\tilde{\boldsymbol{G}}^{r}\right)^{\dagger}$. Here the lead self-energies and lead 
Green functions for the $L$ side are given by

$$
\begin{aligned}
\boldsymbol{\Sigma}_{L}^{r} & =\boldsymbol{t}_{C L} \boldsymbol{g}_{L L}^{r} \boldsymbol{t}_{L C} \\
\boldsymbol{g}_{L L}^{r} & =\left[\left(\epsilon+\mathrm{i} \gamma_{L} / 2\right) \boldsymbol{S}_{L L}-\boldsymbol{H}_{L L}\right]^{-1} \\
\boldsymbol{\Gamma}_{L} & =\mathrm{i}\left(\boldsymbol{\Sigma}_{L}^{r}-\boldsymbol{\Sigma}_{L}^{a}\right) \\
\boldsymbol{\Sigma}_{L}^{+-} & =\boldsymbol{t}_{C L} \boldsymbol{g}_{L L}^{+-} \boldsymbol{t}_{L C}=-\mathrm{i} \boldsymbol{\Gamma}_{L} f_{L} \\
\boldsymbol{\Sigma}_{L}^{-+} & =\boldsymbol{t}_{C L} \boldsymbol{g}_{L L}^{-+} \boldsymbol{t}_{L C}=-\mathrm{i} \boldsymbol{\Gamma}_{L}\left(f_{L}-1\right),
\end{aligned}
$$

where $\boldsymbol{t}_{L C}=\boldsymbol{H}_{L C}-\epsilon \boldsymbol{S}_{L C}$ and so on, with similar expressions for $R$ side. The infinitesimal $\gamma_{C}$ is only needed for recovering the correct result in the limit where the selfenergies are taken to zero - it may be neglected here. The parameters $\gamma_{L, R}$ are positive infinitesimals, which, however, can be used to introduce a finite broadening of the lead eigenstates.

These are enough for calculating the elastic current in the absence of electron-vibration coupling. The full Green functions including the effects of this coupling are

$$
\begin{aligned}
\boldsymbol{G}^{r} & =\left[\epsilon \boldsymbol{S}_{C C}-\boldsymbol{H}_{C C}-\boldsymbol{\Sigma}_{L}^{r}-\boldsymbol{\Sigma}_{R}^{r}-\boldsymbol{\Sigma}_{e-v i b}^{r}\right]^{-1} \\
\boldsymbol{G}^{+-} & =-\boldsymbol{G}^{r}\left(\boldsymbol{\Sigma}_{L}^{+-}+\boldsymbol{\Sigma}_{R}^{+-}+\boldsymbol{\Sigma}_{e-v i b}^{+-}-\mathrm{i} \boldsymbol{\gamma}_{C} f_{C}\right) \boldsymbol{G}^{a} \\
\boldsymbol{G}^{-+} & =-\boldsymbol{G}^{r}\left[\boldsymbol{\Sigma}_{L}^{-+}+\boldsymbol{\Sigma}_{R}^{-+}+\boldsymbol{\Sigma}_{e-v i b}^{-+}-\mathrm{i} \boldsymbol{\gamma}_{C}\left(f_{C}-1\right)\right] \boldsymbol{G}^{a}
\end{aligned}
$$

and $\boldsymbol{G}^{a}=\left(\boldsymbol{G}^{r}\right)^{\dagger}$. To second order in the coupling constant $\boldsymbol{\lambda}^{\alpha}$, the electron-phonon self-energies are

$$
\begin{aligned}
\boldsymbol{\Sigma}_{e-v i b}^{ \pm \mp}(\epsilon)= & -\mathrm{i} \sum_{\alpha} \int \frac{\mathrm{d} \omega_{1}}{2 \pi} D_{\alpha}^{ \pm \mp}\left(\omega_{1}\right)\left[\boldsymbol{\lambda}^{\alpha} \boldsymbol{G}^{ \pm \mp}\left(\epsilon-\omega_{1}\right) \boldsymbol{\lambda}^{\alpha}\right] \\
\boldsymbol{\Sigma}_{e-v i b}^{r}(\epsilon) & =\mathrm{i} \sum_{\alpha} \int \frac{\mathrm{d} \omega_{1}}{2 \pi}\left\{D_{\alpha}^{ \pm \mp}\left(\omega_{1}\right)\left[\boldsymbol{\lambda}^{\alpha} \boldsymbol{G}^{r}\left(\epsilon-\omega_{1}\right) \boldsymbol{\lambda}^{\alpha}\right]\right. \\
& +D_{\alpha}^{r}\left(\omega_{1}\right)\left[\boldsymbol{\lambda}^{\alpha} \boldsymbol{G}^{\mp \pm}\left(\epsilon-\omega_{1}\right) \boldsymbol{\lambda}^{\alpha}\right] \\
& \left.-\boldsymbol{\lambda}^{\alpha} \operatorname{Tr}\left[\boldsymbol{G}^{+-}\left(\omega_{1}\right) \boldsymbol{\lambda}^{\alpha}\right] D_{\alpha}^{r}(0)\right\} \\
\boldsymbol{\Sigma}_{e-v i b}^{a}(\epsilon) & =\mathrm{i} \sum_{\alpha} \int \frac{\mathrm{d} \omega_{1}}{2 \pi}\left\{D_{\alpha}^{ \pm \mp}\left(\omega_{1}\right)\left[\boldsymbol{\lambda}^{\alpha} \boldsymbol{G}^{a}\left(\epsilon-\omega_{1}\right) \boldsymbol{\lambda}^{\alpha}\right]\right. \\
& +D_{\alpha}^{a}\left(\omega_{1}\right)\left[\boldsymbol{\lambda}^{\alpha} \boldsymbol{G}^{\mp \pm}\left(\epsilon-\omega_{1}\right) \boldsymbol{\lambda}^{\alpha}\right] \\
& \left.-\boldsymbol{\lambda}^{\alpha} \operatorname{Tr}\left[\boldsymbol{G}^{+-}\left(\omega_{1}\right) \boldsymbol{\lambda}^{\alpha}\right] D_{\alpha}^{r}(0)\right\},
\end{aligned}
$$

where the upper or lower signs are chosen.

\section{Phonon propagators and self-energies}

By our definition of the phonon propagators, the unperturbed ones (those in the absence of a lead coupling and electron-vibration coupling) are given by

$$
\begin{aligned}
d_{\alpha}^{r}(\epsilon) & =\frac{1}{\epsilon-\epsilon_{\alpha}+\mathrm{i} \eta / 2}-\frac{1}{\epsilon+\epsilon_{\alpha}+\mathrm{i} \eta / 2} \\
& =\frac{2 \epsilon_{\alpha}}{\epsilon^{2}-\epsilon_{\alpha}^{2}+\mathrm{i} \eta \epsilon-\eta^{2} / 4} \\
d_{\alpha}^{+-}(\epsilon) & =-2 \pi \mathrm{i} n(\epsilon) \rho_{\alpha}(\epsilon) \\
d_{\alpha}^{-+}(\epsilon) & =-2 \pi \mathrm{i}[n(\epsilon)+1] \rho_{\alpha}(\epsilon) .
\end{aligned}
$$

Here $\epsilon_{\alpha}=\hbar \omega_{\alpha}$ are the bare vibrational energies, $n(\epsilon)$ is the Bose distribution function. The quantity $\rho_{\alpha}=$ $-\operatorname{Im} d_{\alpha}^{r} / \pi$ is the bare phonon density of states (DOS), given by Eq. (8). This becomes $\rho_{\alpha}(\epsilon)=\delta\left(\epsilon-\epsilon_{\alpha}\right)-\delta(\epsilon+$ $\left.\epsilon_{\alpha}\right)$, as $\eta \rightarrow 0+$. Note that the Green functions satisfy $\eta=\mathrm{i}\left[\left(d^{a}\right)^{-1}-\left(d^{r}\right)^{-1}\right]$.

Now, to be symmetric with the discussion for the electron propagators, the next step should be the introduction propagators " $\tilde{D}$ " which contain lead self-energies. For the proper calculation of the lead self-energies, we should probably change to an atomic-displacement basis. Instead of doing this, we model the lead coupling by giving finite values to the infinitesimal quantity $\eta$, which will broaden the phonon density of states 31].

The full phonon propagators appearing in the electronphonon self-energies are obtained from the "Dyson" and "Keldysh" equations for phonons

$$
\begin{aligned}
D_{\alpha}^{r} & =\left[\left(d_{\alpha}^{r}\right)^{-1}-\Pi_{\alpha}^{r}\right]^{-1} \\
D_{\alpha}^{ \pm \mp} & =\left(1+D_{\alpha}^{r} \Pi_{\alpha}^{r}\right) d_{\alpha}^{ \pm \mp}\left(1+\Pi_{\alpha}^{a} D_{\alpha}^{a}\right)-D_{\alpha}^{r} \Pi_{\alpha}^{ \pm \mp} D_{\alpha}^{a} .
\end{aligned}
$$

For self-energies, or "polarizations", we use the secondorder approximations

$$
\begin{aligned}
\Pi_{\alpha}^{ \pm \mp}(\epsilon) & =\mathrm{i} \int \frac{\mathrm{d} \omega_{1}}{2 \pi} \operatorname{Tr}\left[\boldsymbol{\lambda}^{\alpha} \boldsymbol{G}^{ \pm \mp}\left(\omega_{1}\right) \boldsymbol{\lambda}^{\alpha} \boldsymbol{G}^{\mp \pm}\left(\omega_{1}-\epsilon\right)\right] \\
\Pi_{\alpha}^{r}(\epsilon) & =-\mathrm{i} \int \frac{\mathrm{d} \omega_{1}}{2 \pi} \operatorname{Tr}\left[\boldsymbol{\lambda}^{\alpha} \boldsymbol{G}^{ \pm \mp}\left(\omega_{1}\right) \boldsymbol{\lambda}^{\alpha} \boldsymbol{G}^{a}\left(\omega_{1}-\epsilon\right)\right. \\
& \left.+\boldsymbol{\lambda}^{\alpha} \boldsymbol{G}^{r}\left(\omega_{1}\right) \boldsymbol{\lambda}^{\alpha} \boldsymbol{G}^{ \pm \mp}\left(\omega_{1}-\epsilon\right)\right] \\
\Pi_{\alpha}^{a}(\epsilon) & =-\mathrm{i} \int \frac{\mathrm{d} \omega_{1}}{2 \pi} \operatorname{Tr}\left[\boldsymbol{\lambda}^{\alpha} \boldsymbol{G}^{ \pm \mp}\left(\omega_{1}\right) \boldsymbol{\lambda}^{\alpha} \boldsymbol{G}^{r}\left(\omega_{1}-\epsilon\right)\right. \\
& \left.+\boldsymbol{\lambda}^{\alpha} \boldsymbol{G}^{a}\left(\omega_{1}\right) \boldsymbol{\lambda}^{\alpha} \boldsymbol{G}^{ \pm \mp}\left(\omega_{1}-\epsilon\right)\right],
\end{aligned}
$$

where we have dropped some unimportant zero-frequency terms. Again either the upper or the lower signs must be chosen. Note that $\Pi_{\alpha}^{ \pm \mp}$ are purely imaginary and satisfy the symmetry $\Pi_{\alpha}^{+-}(-\epsilon)=\Pi_{\alpha}^{-+}(\epsilon)$.

Equations (C10) close the system of equations, and we are done. However, from a physical point of view, it is interesting to develop the equations slightly further. Using the symmetry $D_{\alpha}^{r}-D_{\alpha}^{a}=D_{\alpha}^{-+}-D_{\alpha}^{+-}$, one finds that Eqs. (C9) may be rewritten in the form

$$
\begin{aligned}
D_{\alpha}^{r}(\epsilon) & =\frac{2 \epsilon_{\alpha}}{\epsilon^{2}-\epsilon_{\alpha}^{2}+\mathrm{i} \eta \epsilon-\eta^{2} / 4-2 \epsilon_{\alpha} \Pi_{\alpha}^{r}(\epsilon)} \\
D_{\alpha}^{+-}(\epsilon) & =-2 \pi \mathrm{i} N_{\alpha}(\epsilon) \rho_{\alpha}(\epsilon) \\
D_{\alpha}^{-+}(\epsilon) & =-2 \pi \mathrm{i}\left(N_{\alpha}(\epsilon)+1\right) \rho_{\alpha}(\epsilon),
\end{aligned}
$$

where we define the phonon density of states

$$
\rho_{\alpha}(\epsilon)=-\frac{1}{\pi} \operatorname{Im} D_{\alpha}^{r}(\epsilon),
$$

which satisfies $\rho_{\alpha}(-\epsilon)=-\rho_{\alpha}(\epsilon)$. The quantity $N_{\alpha}(\epsilon)$ is the energy distribution function of the vibrational 
quanta. In equilibrium $N_{\alpha}(\epsilon)=n(\epsilon)$, the Bose distribution. In general $N_{\alpha}(\epsilon)=n(\epsilon)+\delta N_{\alpha}(\epsilon)$, where $\delta N_{\alpha}(\epsilon)$ is a voltage-dependent non-equilibrium correction.

By reshuffling the Keldysh equations one may write

$$
\begin{aligned}
& D_{\alpha}^{+-}(\epsilon)=-D_{\alpha}^{r}\left[\mathrm{i} n \epsilon \eta / \epsilon_{\alpha}+\Pi_{\alpha}^{+-}\right] D_{\alpha}^{a} \\
& D_{\alpha}^{-+}(\epsilon)=-D_{\alpha}^{r}\left[\mathrm{i}(1+n) \epsilon \eta / \epsilon_{\alpha}+\Pi_{\alpha}^{-+}\right] D_{\alpha}^{a} .
\end{aligned}
$$

Since $\left|D_{\alpha}^{r}\right|^{2}=\operatorname{Im} D_{\alpha}^{r} /\left(\operatorname{Im} \Pi_{\alpha}^{r}(\epsilon)-\eta \epsilon / 2 \epsilon_{\alpha}\right)$, comparing these with Eqs. (C11) it is easy to obtain explicit expressions for the distribution function $N_{\alpha}$ :

$$
\begin{aligned}
N_{\alpha}(\epsilon) & =-\frac{1}{2} \frac{\operatorname{Im} \Pi_{\alpha}^{+-}(\epsilon)+n(\epsilon) \eta \epsilon / \epsilon_{\alpha}}{\operatorname{Im} \Pi_{\alpha}^{r}(\epsilon)-\eta \epsilon / 2 \epsilon_{\alpha}} \\
& =-\frac{1}{2} \frac{\operatorname{Im} \Pi_{\alpha}^{-+}(\epsilon)+[1+n(\epsilon)] \eta \epsilon / \epsilon_{\alpha}}{\operatorname{Im} \Pi_{\alpha}^{r}(\epsilon)-\eta \epsilon / 2 \epsilon_{\alpha}}-1 .
\end{aligned}
$$

To get to the last line, we used $\Pi_{\alpha}^{r}-\Pi_{\alpha}^{a}=-\left(\Pi_{\alpha}^{-+}-\right.$
$\left.\Pi_{\alpha}^{+-}\right)$. Note that we have not made any approximations to get to this result. Using Eqs. [C10, one finds that the following symmetries are valid: $N_{\alpha}(-\epsilon)=-\left[N_{\alpha}(\epsilon)+1\right]$ and $\delta N_{\alpha}(-\epsilon)=-\delta N_{\alpha}(\epsilon)$.

\section{APPENDIX D: CURRENT CONSERVATION}

The inelastic parts of the current in Eqs. (4) look very asymmetric in their $L$ or $R$ indices. Nevertheless, in stationary state the currents calculated at $L$ and $R$ boundaries should be equal: $I^{L}=I^{R}$. There also appears to be some confusion as to what sort of approximations are needed for current conservation [19, 36]. Here we outline a proof of this property for our approximation. For $I_{e l}$ it is obvious, so we only consider the inelastic current.

Inserting the self-energies from Eq. (C7) into the expression for $I_{\text {inel }}^{L}$ [Eq. (3)] $]$, one finds

$$
\begin{aligned}
I_{\text {inel }}^{L}= & \frac{2 e}{\hbar} \sum_{\alpha} \int \frac{\mathrm{d} \epsilon}{2 \pi} \int \frac{\mathrm{d} \omega_{1}}{2 \pi} 2 \pi \rho_{\alpha}\left(\omega_{1}\right)\{ \\
& \operatorname{Tr}\left[\boldsymbol{G}^{a}\left(\epsilon-\frac{\omega_{1}}{2}\right) \boldsymbol{\lambda}^{\alpha} \boldsymbol{G}^{a}\left(\epsilon+\frac{\omega_{1}}{2}\right) \boldsymbol{\Gamma}_{L}\left(\epsilon+\frac{\omega_{1}}{2}\right) \boldsymbol{G}^{r}\left(\epsilon+\frac{\omega_{1}}{2}\right) \boldsymbol{\lambda}^{\alpha} \boldsymbol{G}^{r}\left(\epsilon-\frac{\omega_{1}}{2}\right) \boldsymbol{\Gamma}_{L}\left(\epsilon-\frac{\omega_{1}}{2}\right)\right] \\
& \times\left[f_{L}\left(\epsilon+\frac{\omega_{1}}{2}\right)\left(N_{\alpha}\left(\omega_{1}\right)+1\right)-\left(f_{L}\left(\epsilon+\frac{\omega_{1}}{2}\right)+N_{\alpha}\left(\omega_{1}\right)\right) f_{L}\left(\epsilon-\frac{\omega_{1}}{2}\right)\right] \\
+ & \operatorname{Tr}\left[\boldsymbol{G}^{a}\left(\epsilon-\frac{\omega_{1}}{2}\right) \boldsymbol{\lambda}^{\alpha} \boldsymbol{G}^{a}\left(\epsilon+\frac{\omega_{1}}{2}\right) \boldsymbol{\Gamma}_{L}\left(\epsilon+\frac{\omega_{1}}{2}\right) \boldsymbol{G}^{r}\left(\epsilon+\frac{\omega_{1}}{2}\right) \boldsymbol{\lambda}^{\alpha} \boldsymbol{G}^{r}\left(\epsilon-\frac{\omega_{1}}{2}\right) \boldsymbol{\Gamma}_{R}\left(\epsilon-\frac{\omega_{1}}{2}\right)\right] \\
& \left.\times\left[f_{L}\left(\epsilon+\frac{\omega_{1}}{2}\right)\left(N_{\alpha}\left(\omega_{1}\right)+1\right)-\left(f_{L}\left(\epsilon+\frac{\omega_{1}}{2}\right)+N_{\alpha}\left(\omega_{1}\right)\right) f_{R}\left(\epsilon-\frac{\omega_{1}}{2}\right)\right]\right\} .
\end{aligned}
$$

Here the first term may be shown to vanish as follows. Since Fermi and Bose functions $f$ and $n$ satisfy $f(x)[n(y)+1]-[f(x)+n(y)] f(x-y)=0$, we have

$$
\begin{gathered}
f_{L}(x)\left[N_{\alpha}(y)+1\right]-\left[f_{L}(x)+N_{\alpha}(y)\right] f_{L}(x-y) \\
=\delta N_{\alpha}(y)\left[f_{L}(x)-f_{L}(x-y)\right] .
\end{gathered}
$$

Now, noting that $\delta N_{\alpha}(-\epsilon)=-\delta N_{\alpha}(\epsilon)$, and $\rho_{\alpha}(-\epsilon)=$ $-\rho_{\alpha}(\epsilon)$, and additionally assuming that all matrices under the trace are symmetric $\left(\boldsymbol{G}^{r T}=\boldsymbol{G}^{r},\left[\boldsymbol{\Gamma}_{L}\right]^{T}=\boldsymbol{\Gamma}_{L}\right.$ etc.) it is seen that the integrand is odd and thus the energy integral vanishes. This is true also if $\delta N_{\alpha}(\epsilon)$ has a $\sim 1 / \epsilon$ divergence at $\epsilon=0$, since the product in Eq. (D2) remains finite.

The second term in Eq. (D1) is clearly symmetric upon interchanging $L$ and $R$, and changing the overall sign. Note that the proof does not rely on things like a mirror symmetry of the geometry, and remains unchanged if we replace $\boldsymbol{G}^{r} \rightarrow \tilde{\boldsymbol{G}}^{r}$. Thus our expressions are always "current conserving", as defined by the condition $I^{L}=I^{R}$.

\section{APPENDIX E: GENERAL PERTURBATIVE CURRENT FORMULAS}

Here we shall write down the perturbative current formulas of Eqs. (15) in a slightly different form, which will make the so-called wide-band approximation more transparent. However, here we shall be making no approximations in addition to the second-order perturbation theory which we have already introduced. Nevertheless, it must be stressed that, due to the second-order approximation, all higher-order terms in the following expressions are strictly speaking not warranted. This concerns the approximations made for the vibrational density of states $\rho_{\alpha}$ and the distribution $N_{\alpha}$, which should in principle both be of zeroth order in the electron-vibration coupling constant. In the approximations which we use for $N_{\alpha}$, this is not necessarily the case. Thus our approach is not purely lowest-oder perturbation theory. However, the self-consistent Born scheme (SCBA) is not really any better is this respect.

The inelastic current $I_{i n}$, and the elastic parts $I_{e l}^{0}$ and $\delta I_{e l}$ are obtained from Eqs. (5). Inserting the selfenergies [Eqs. [C7] ] into these formulas, they may be 
rewritten as follows:

$$
\begin{gathered}
I_{e l}^{0}=\frac{2 e}{h} \int \mathrm{d} \epsilon T_{0}(\epsilon)\left[f_{L}(\epsilon)-f_{R}(\epsilon)\right] \\
\delta I_{e l}=\frac{2 e}{h} \int \mathrm{d} \epsilon \sum_{\alpha} \sum_{\sigma= \pm 1} \sigma \int_{0}^{\infty} \mathrm{d} \omega_{1} \rho_{\alpha}\left(\omega_{1}\right)\left\{T_{\sigma \alpha}^{e c}\left(\epsilon, \omega_{1}\right) N_{\alpha}\left(\sigma \omega_{1}\right)+T_{\sigma \alpha}^{e c L}\left(\epsilon, \omega_{1}\right) f_{L}\left(\epsilon_{\sigma \alpha}\right)+T_{\sigma \alpha}^{e c R}\left(\epsilon, \omega_{1}\right) f_{R}\left(\epsilon_{\sigma \alpha}\right)\right\} \\
\times\left[f_{L}(\epsilon)-f_{R}(\epsilon)\right]+\frac{2 e}{h} \int \mathrm{d} \epsilon \sum_{\alpha}\left\{-J_{\alpha}^{L}(\epsilon)-J_{\alpha}^{R}(\epsilon)+T_{\alpha}^{I I}(\epsilon)\left[J_{\alpha}^{I I L}+J_{\alpha}^{I I R}\right]\right\}\left[f_{L}(\epsilon)-f_{R}(\epsilon)\right] \\
I_{\text {inel }}^{L}=\frac{2 e}{h} \int \mathrm{d} \epsilon \sum_{\alpha} \sum_{\sigma= \pm 1} \sigma \int_{0}^{\infty} \mathrm{d} \omega_{1} \rho_{\alpha}\left(\omega_{1}\right) T_{\sigma \alpha}^{i n}\left(\epsilon, \omega_{1}\right)\left\{N_{\alpha}\left(\sigma \omega_{1}\right) f_{L}(\epsilon)\left[1-f_{R}\left(\epsilon_{\sigma \alpha}\right)\right]\right. \\
\left.+N_{\alpha}\left(-\sigma \omega_{1}\right) f_{R}\left(\epsilon_{\sigma \alpha}\right)\left[1-f_{L}(\epsilon)\right]\right\}
\end{gathered}
$$

where $\epsilon_{\sigma \alpha}=\epsilon+\sigma \omega_{1}$. The elastic transmission is given by

$$
T_{0}(\epsilon)=\operatorname{Tr}\left[\tilde{\boldsymbol{G}}^{r}(\epsilon) \boldsymbol{\Gamma}_{R}(\epsilon) \tilde{\boldsymbol{G}}^{a}(\epsilon) \boldsymbol{\Gamma}_{L}(\epsilon)\right]
$$

and the inelastic prefactor by

$$
T_{\sigma \alpha}^{i n}\left(\epsilon, \omega_{1}\right)=\operatorname{Tr}\left[\tilde{\boldsymbol{G}}^{r}\left(\epsilon_{\sigma \alpha}\right) \boldsymbol{\Gamma}_{R}\left(\epsilon_{\sigma \alpha}\right) \tilde{\boldsymbol{G}}^{a}\left(\epsilon_{\sigma \alpha}\right) \boldsymbol{\lambda}^{\alpha} \tilde{\boldsymbol{G}}^{a}(\epsilon) \boldsymbol{\Gamma}_{L}(\epsilon) \tilde{\boldsymbol{G}}^{r}(\epsilon) \boldsymbol{\lambda}^{\alpha}\right]
$$

while the factors in the elastic correction are

$$
\begin{aligned}
T_{\sigma \alpha}^{e c}\left(\epsilon, \omega_{1}\right) & =2 \operatorname{Re} \operatorname{Tr}\left[\tilde{\boldsymbol{G}}^{r}(\epsilon) \boldsymbol{\Gamma}_{R}(\epsilon) \tilde{\boldsymbol{G}}^{a}(\epsilon) \boldsymbol{\Gamma}_{L}(\epsilon) \tilde{\boldsymbol{G}}^{r}(\epsilon) \boldsymbol{\lambda}^{\alpha} \tilde{\boldsymbol{G}}^{r}\left(\epsilon_{\sigma \alpha}\right) \boldsymbol{\lambda}^{\alpha}\right] \\
T_{\sigma \alpha}^{e c L}\left(\epsilon, \omega_{1}\right) & =\operatorname{Im} \operatorname{Tr}\left[\tilde{\boldsymbol{G}}^{r}(\epsilon) \boldsymbol{\Gamma}_{R}(\epsilon) \tilde{\boldsymbol{G}}^{a}(\epsilon) \boldsymbol{\Gamma}_{L}(\epsilon) \tilde{\boldsymbol{G}}^{r}(\epsilon) \boldsymbol{\lambda}^{\alpha} \tilde{\boldsymbol{G}}^{r}\left(\epsilon_{\sigma \alpha}\right) \boldsymbol{\Gamma}_{L}\left(\epsilon_{\sigma \alpha}\right) \tilde{\boldsymbol{G}}^{a}\left(\epsilon_{\sigma \alpha}\right) \boldsymbol{\lambda}^{\alpha}\right] \\
T_{\sigma \alpha}^{e c R}\left(\epsilon, \omega_{1}\right) & =\operatorname{Im} \operatorname{Tr}\left[\tilde{\boldsymbol{G}}^{r}(\epsilon) \boldsymbol{\Gamma}_{R}(\epsilon) \tilde{\boldsymbol{G}}^{a}(\epsilon) \boldsymbol{\Gamma}_{L}(\epsilon) \tilde{\boldsymbol{G}}^{r}(\epsilon) \boldsymbol{\lambda}^{\alpha} \tilde{\boldsymbol{G}}^{r}\left(\epsilon_{\sigma \alpha}\right) \boldsymbol{\Gamma}_{R}\left(\epsilon_{\sigma \alpha}\right) \tilde{\boldsymbol{G}}^{a}\left(\epsilon_{\sigma \alpha}\right) \boldsymbol{\lambda}^{\alpha}\right]
\end{aligned}
$$

The most complicated part are the integrals

$$
\begin{aligned}
& J_{\alpha}^{L}(\epsilon)=\int \frac{\mathrm{d} \omega_{1}}{2 \pi} 2 \operatorname{Re}\left[D_{\alpha}^{r}\left(\omega_{1}\right)\right] \operatorname{Re} \operatorname{Tr}\left[\tilde{\boldsymbol{G}}^{r}(\epsilon) \boldsymbol{\Gamma}_{R}(\epsilon) \tilde{\boldsymbol{G}}^{a}(\epsilon) \boldsymbol{\Gamma}_{L}(\epsilon) \tilde{\boldsymbol{G}}^{r}(\epsilon) \boldsymbol{\lambda}^{\alpha} \tilde{\boldsymbol{G}}^{r}\left(\epsilon-\omega_{1}\right) \boldsymbol{\Gamma}_{L}\left(\epsilon-\omega_{1}\right) \tilde{\boldsymbol{G}}^{a}\left(\epsilon-\omega_{1}\right) \boldsymbol{\lambda}^{\alpha}\right] f_{L}\left(\epsilon-\omega_{1}\right) \\
& J_{\alpha}^{R}(\epsilon)=\int \frac{\mathrm{d} \omega_{1}}{2 \pi} 2 \operatorname{Re}\left[D_{\alpha}^{r}\left(\omega_{1}\right)\right] \operatorname{Re} \operatorname{Tr}\left[\tilde{\boldsymbol{G}}^{r}(\epsilon) \boldsymbol{\Gamma}_{R}(\epsilon) \tilde{\boldsymbol{G}}^{a}(\epsilon) \boldsymbol{\Gamma}_{L}(\epsilon) \tilde{\boldsymbol{G}}^{r}(\epsilon) \boldsymbol{\lambda}^{\alpha} \tilde{\boldsymbol{G}}^{r}\left(\epsilon-\omega_{1}\right) \boldsymbol{\Gamma}_{R}\left(\epsilon-\omega_{1}\right) \tilde{\boldsymbol{G}}^{a}\left(\epsilon-\omega_{1}\right) \boldsymbol{\lambda}^{\alpha}\right] f_{R}\left(\epsilon-\omega_{1}\right)
\end{aligned}
$$

and the coefficients coming from the last term in Eq. (C7) for $\boldsymbol{\Sigma}^{r}$

$$
\begin{aligned}
T_{\alpha}^{I I}(\epsilon) & =2 \operatorname{Re} \operatorname{Tr}\left[\tilde{\boldsymbol{G}}^{r}(\epsilon) \boldsymbol{\Gamma}_{R}(\epsilon) \tilde{\boldsymbol{G}}^{a}(\epsilon) \boldsymbol{\Gamma}_{L}(\epsilon) \tilde{\boldsymbol{G}}^{r}(\epsilon) \boldsymbol{\lambda}^{\alpha}\right] \\
J_{\alpha}^{I I L} & =D_{\alpha}^{r}(0) \int \frac{\mathrm{d} \omega_{1}}{2 \pi} \operatorname{Tr}\left[\tilde{\boldsymbol{G}}^{r}\left(\omega_{1}\right) \boldsymbol{\Gamma}_{L}\left(\omega_{1}\right) \tilde{\boldsymbol{G}}^{a}\left(\omega_{1}\right) \boldsymbol{\lambda}^{\alpha}\right] f_{L}\left(\omega_{1}\right) \\
J_{\alpha}^{I I R} & =D_{\alpha}^{r}(0) \int \frac{\mathrm{d} \omega_{1}}{2 \pi} \operatorname{Tr}\left[\tilde{\boldsymbol{G}}^{r}\left(\omega_{1}\right) \boldsymbol{\Gamma}_{R}\left(\omega_{1}\right) \tilde{\boldsymbol{G}}^{a}\left(\omega_{1}\right) \boldsymbol{\lambda}^{\alpha}\right] f_{R}\left(\omega_{1}\right) .
\end{aligned}
$$

In the equations above, the phonon density of states $\rho_{\alpha}(\epsilon)$ includes all possible broadening effects and shifts of the bare vibrational frequencies. Without these effects $\rho_{\alpha}(\epsilon)=\delta\left(\epsilon-\hbar \omega_{\alpha}\right)-\delta\left(\epsilon+\hbar \omega_{\alpha}\right)$, and Eqs. (E7) must be evaluated as principal part integrals.

In the so-called wide band limit, discussed in the text, the $\epsilon$ and $\omega_{1}$ dependences of the coefficients $T_{0}, T_{\alpha}^{i n}, T_{\alpha}^{e c}$ and $T_{\alpha}^{e c L, R}$, may be dropped and they may be simply 
evaluated at the Fermi energy. Then the integrals over products of Fermi functions in the corresponding current terms can be done analytically. One also finds that all terms of $\delta I_{e l}$ which do not involve Eqs. (E7) or E8) yield conductance contributions $\delta G_{e l}(\epsilon)=\mathrm{d} \delta I_{e l} / \mathrm{d} V$ which are symmetric in the bias: $\delta G_{e l}(-V)=\delta G_{e l}(V)$. We call the sum of these terms $\delta I_{e l}^{s y m}$, and $I^{s y m}=I_{e l}^{0}+\delta I_{e l}^{\text {sym }}+I_{\text {inel }}$. The same may be done to the trace expressions in Eqs. (E7), and one finds that the corresponding part in the current $\delta I_{e l}$ yields the asymmetric current $I^{\text {asy }}$ 36].
The contribution of Eqs. (E8) to the current is typically very small in the small-voltage limit which we are considering. Furthermore, since they do not introduce any relation between the voltage and the vibrational frequencies, they cannot give a contribution to the conductance steps. Therefore, we drop them for the sake of simplicity.
[1] N. Agraï, A. L. Yeyati, J. M. van Ruitenbeek, Phys. Rep. 377, 81 (2003).

[2] E. Scheer, N. Agrait, J. C. Cuevas, A. L. Yeyati, B. Ludolph, A. Martin-Rodero, G. R. Bollinger, J. M. van Ruitenbeek, C. Urbina, Nature 394, 154 (1998).

[3] H. Ohnishi, Y. Kondo, and K. Takayanagi, Nature (London) 395, 780 (1998).

[4] A. I. Yanson, G. Rubio-Bollinger, H. E. van den Brom, N. Agrait, and J. M. van Ruitenbeek, Nature (London) 395, 783 (1998).

[5] R. H. M. Smit, C. Untiedt, A. I. Yanson, and J. M. van Ruitenbeek, Phys. Rev. Lett. 87, 266102 (2001).

[6] C. Untiedt, A. I. Yanson, R. Grande, G. Rubio-Bollinger, N. Agraït, S. Vieira, and J. M. van Ruitenbeek, Phys. Rev. B 66, 085418 (2002).

[7] R. H. M. Smit, C. Untiedt, G. Rubio-Bollinger, R. C. Segers, and J. M. van Ruitenbeek, Phys. Rev. Lett. 91, 076805 (2003).

[8] E.G. Emberly and G. Kirczenow, Phys. Rev. B 60, 6028 (1999).

[9] M. Brandbyge, J.-L. Mozos, P. Ordejón, J. Taylor, and K. Stokbro, Phys. Rev. B 65, 165401 (2002).

[10] J.J. Palacios, A.J. Pérez-Jiménez, E. Louis, E. SanFabián, and J.A. Vergés, Phys. Rev. B 66, 035322 (2002).

[11] M. Zhuang and M. Ernzerhof, J. Chem. Phys. 120, 4921 (2004).

[12] Y. J. Lee, M. Brandbyge, M. J. Puska, J. Taylor, K. Stokbro, and R. M. Nieminen Phys. Rev. B 69, 125409 (2004).

[13] L. de la Vega, A. Martín-Rodero, A. L. Yeyati, and A. Saúl, Phys. Rev. B 70, 113107 (2004).

[14] B. Ludoph, M. H. Devoret, D. Esteve, C. Urbina, and J. M. van Ruitenbeek, Phys. Rev. Lett. 82, 1530 (1999).

[15] E. Scheer, W. Belzig, Y. Naveh, M. H. Devoret, D. Esteve, and C. Urbina, Phys. Rev. Lett. 86, 284 (2001).

[16] G. Rubio-Bollinger, C. de las Heras, E. Bascones, N. Agraï, F. Guinea, and S. Vieira, Phys. Rev. B 67, 121407(R) (2003).

[17] S. K. Nielsen, M. Brandbyge, K. Hansen, K. Stokbro, J. M. van Ruitenbeek, and F. Besenbacher, Phys. Rev. Lett. 89, 066804 (2002).

[18] N. Agrait, C. Untiedt, G. Rubio-Bollinger, and S. Vieira, Phys. Rev. Lett. 88, 216803 (2002).

[19] T. Frederiksen, M. Brandbyge, N. Lorente, and A.-P. Jauho, Phys. Rev. Lett. 93, 256601 (2004).

[20] T. Frederiksen, M. Brandbyge, A.-P. Jauho, and N. Lorente, cond-mat/0411108
[21] J. C. Slater and G. F. Koster, Physical Review 94, 1498 (1954).

[22] R. E. Cohen, M. J. Mehl, and D. A. Papaconstantopoulos, Phys. Rev. B 50, R14694 (1994).

[23] M. J. Mehl and D. A. Papaconstantopoulos, Phys. Rev. B 54, 4519 (1996).

[24] D. A. Papaconstantopoulos and M. J. Mehl, J. Phys. Condens. Matter 15, R413 (2003).

[25] http://cst-www.nrl.navy.mil/bind/au_par_99.

[26] M. J. Montgomery, J. Hoekstra, T. N. Todorov and A. P. Sutton, J. Phys. Condens. Matter 15, 731 (2003).

[27] E. G. Emberly and G. Kirczenow, Phys. Rev. B 61, 5740 (2000).

[28] M. Brandbyge, N. Kobayashi, M. Tsukada, Phys. Rev. B 60, 17064 (1999).

[29] T. Mii, S. G. Tikhodeev, and H. Ueba, Phys. Rev. B 68, 205406 (2003).

[30] A. Mitra, I. Aleiner, and A. J. Millis, Phys. Rev. B 69, 245302 (2004).

[31] M. Galperin, M.A. Ratner, and A. Nitzan, J. Chem. Phys. 121, 11965 (2004).

[32] G. Mahan, Many-Particle Physics, 2nd ed. (Plenum, New York, 1990).

[33] Y. Chen, M. Zwolak, and M. Di Ventra, Nano Lett. 3, 1691 (2003).

[34] F. Guinea, C. Tejedor, F. Flores, and E. Louis, Phys. Rev. B 28, 4397 (1983).

[35] Y. Chen, M. Di Ventra, cond-mat/0505005

[36] M. Paulsson, T. Frederiksen, and M. Brandbyge, cond-mat/0505473

[37] W. P. Su, J. R. Schrieffer, and A. J. Heeger, Phys. Rev. Lett. 42, 1698 (1979).

[38] D. Sánchez-Portal, E. Artacho, J. Junquera, P. Ordejón, A. García, J. M. Soler, Phys. Rev. Lett. 83, 3884 (1999).

[39] E. Z. da Silva, F. D. Novaes, A. J. R. da Silva, and A. Fazzio, Phys. Rev. B 69, 115411 (2004).

[40] M. Dreher, F. Pauly, J. Heurich, J.C. Cuevas, E. Scheer, P. Nielaba, cond-mat/0406281

[41] M. Head-Gordon and J. C. Tully, J. Chem. Phys. 96, 3939 (1991).

[42] Y. Xue, S. Datta, and M. A. Ratner, Chem. Phys. 281, $151(2002)$.

[43] A. Szabo and N. S. Ostlund, Modern Quantum Chemistry, (Dover, New York, 1982).

[44] E. Emberly and G. Kirczenow, Phys. Rev. Lett. 81, 5205 (1998). 